\title{
Pure bending of Pipe-in-pipe Systems
}

\author{
Ali Binazir ${ }^{1}$, Hassan Karampour ${ }^{1, *}$ Adam J. Sadowski ${ }^{2}$, and Benoit P. Gilbert ${ }^{1}$ \\ ${ }^{1}$ Griffith School of Engineering and Built Environment, Griffith University, Gold Coast \\ Campus, QLD 4222, Australia \\ ${ }^{2}$ Department of Civil and Environmental Engineering, Imperial College London, UK
}

\begin{abstract}
Subsea pipelines and PIP systems experience large bending moments during installation and operation. However, unlike single-walled pipelines, the behaviour of PIPs under bending has been only marginally addressed. In the current study, the bending response of PIP systems with diameter-to-thickness ratio $(D / t)$ of 15 to 40 is investigated. Linear bifurcation analyses (LBA) and geometrically nonlinear analyses (GNA) are conducted on PIPs of varying lengths. Analytical expressions are provided to predict the classical and nonlinear limit moments of PIPs, and are compared to existing expressions for single-walled pipelines. Ultimate bending moments of PIPs are obtained from physical four-point bending tests and are compared against geometrically and materially nonlinear analyses (GMNA). The finite element results show that in PIPs with centralizers, the limit moments (GNA) drop slightly, however, the ultimate moments (GMNA) remain unchanged. A parametric study of the effect of geometry and material properties of the inner and outer pipes on the ultimate moment of PIPs is presented. It is understood that the ultimate moments of PIPs with thick tubes are predominantly influenced by the material nonlinearities rather than ovalization of the tubes.
\end{abstract}




\section{Nomenclature}

D Mean diameter

$D_{\max } \quad$ Maximum mean diameter

$D_{\text {min }} \quad$ Minimum mean diameter

E Modulus of elasticity

$E^{\prime} \quad$ Tangent modulus

$f_{0} \quad$ Ovalization

$\kappa \quad$ Curvature (mean cross-sectional curvature assuming plane sections are plane)

$\kappa_{c} \quad$ Classical critical curvature (mean cross-sectional curvature)

$\kappa_{\mathrm{Br}} \quad$ Curvature at Brazier moment (mean cross-sectional curvature)

$\lambda \quad$ Linear meridional bending half-wavelength

$M_{c l} \quad$ Classical elastic buckling moment

$M_{c r} \quad$ Critical buckling moment

$M_{B r} \quad$ Brazier moment

$M_{P} \quad$ Plastic moment

$M_{\text {LBA }}$ Linear bifurcation buckling moment

$M_{G N A}$ Elastic non-linear buckling moment

$M_{\text {MNA }}$ Ultimate plastic collapse moment

$M_{\text {GMNA }}$ Elastic-plastic nonlinear buckling moment

$\Omega \quad$ Dimensionless length group

$r \quad$ Mean outside radius

t Pipe wall-thickness

$U \quad$ Total strain energy per unit length of the pipeline

$v \quad$ Poisson's ratio

$\sigma_{y} \quad$ Yield stress

$\sigma_{c r} \quad$ Critical buckling stress

$\omega \quad$ Dimensionless length group

w Radial displacement of the cross section

$v \quad$ Tangential displacement of the cross section

$\delta_{0} \quad$ Maximum amplitude of initial imperfection 


\section{Introduction}

A subsea pipe-in-pipe (PIP) system is comprised of an inner pipe (a flowline), which transports oil or gas with high temperature (HT) \& pressure (HP) and an outer pipe (carrier) which provides mechanical protection from external damage and resists the hydrostatic pressure. The annulus (the space between the tubes) is either empty or filled with a non-structural insulation material such as aerogel. Nylong rings (centralizers) are positioned at regular intervals in order to protect the thermal insulation and to maintain concentricity of the outer and inner tubes during assembly, installation and operation. PIPs are preferred to conventional single-walled pipelines in deep waters due to their superior thermal insulation. In such environments, the hydrostatic pressure resisted by the outer pipe can be as large as $30 \mathrm{MPa}$ (water depths up to 3,000 $\mathrm{m}$ ) and the temperature and internal pressure of the hydrocarbons inside the inner pipe can be as high as $180^{\circ} \mathrm{C}$ and $10 \mathrm{MPa}$ respectively [1].

The subsea pipelines or pipe-in-pipe systems experience axial loads, hydrostatic pressure, internal pressure, bending and combination of these actions during their installation and operation life [2,3]. Axial compressive loads develop due to the seabed resisting the expansion of the pipeline caused by the high pressure and high temperature (HP/HT) of the transmitted fluids. At large axial compressive forces, HP/HT flow can cause global upheaval buckling [4] or lateral buckling $[5,6]$ of the pipeline or the PIP system. The pipeline or the PIP system may undergo large bending moments during installation using S-Lay, J-Lay and the reel methods [7], or during operation, upheaval and lateral buckling [8]. The external pressure during installation, operation and shut-down periods can cause collapse of the carrier pipe [9] and if the pressure is maintained it may also cause collapse of the inner pipe [10] and/or the propagation buckling in the PIP system [11-14]. Subsea pipelines may collapse under combined actions of bending, axial force and external pressure [15]. 
The structural response of a single cylinder under bending has been extensively studied in the past, most notably are the work done by [16-21, 36]. Previous studies have shown that, thin cylinders (large $D / t$ ) subjected to bending may exercise wrinkling and shell-type buckling which eventually results in a localised collapse [19]. This type of failure, known as bifurcation buckling, is predominantly elastic. Knowing that the critical buckling stress of a cylinder under uniaxial compression with modulus of Elasticity $E$, Poisson's ratiov, wall-thickness $t$, and mid-surface radius $r$, is [22].

$$
\sigma_{c l}=\frac{E t}{r \sqrt{3\left(1-v^{2}\right)}}
$$

Seide and Weingarten [20] showed that short cylinders with large $D / t$ ratios ( $D$ is the midsurface diameter) would buckle under bending once the first finite portion of the section reaches the classical buckling stress of the same cylinder under uniform axial compression (Eq. 1). The classical elastic buckling moment $M_{c l}$ of a tube under bending can then be calculated from [20], where it is assumed that local buckling occurs when the extreme fibre stress reaches

$$
\begin{aligned}
& \sigma_{c l} . \\
& M_{c l}=\pi r^{2} t \sigma_{c l}=\frac{E \pi r t^{2}}{\sqrt{3\left(1-v^{2}\right)}}
\end{aligned}
$$

Using a shell theory [23] and considering a linear pre-buckling membrane stress state with no geometric nonlinearities, Seide and Weingarten [20] investigated bending of short tubes with length-to-diameter $(L / D)$ ratio of less than 0.15 , and reported the development and growth of short wavelength wrinkles in the longitudinal direction of the tubes with large $D / t$. They observed that the depth of such wrinkles increases with the corresponding increase in the bending curvature, until the section collapses catastrophically at a moment, known as the bifurcation buckling moment. 
Another type of failure belongs to the thick to moderately thick pipes with $D / t<40$. This groups of tubes may exhibit a limit moment due to the cross-sectional flattening known as ovalization [24], or the combination of bifurcation buckling and ovalization depending on the material and geometrical properties. Studies conducted by Brazier [24] on long single tubes aimed to explain the difference between the bending response of the tubes observed in the physical tests, and expectations from the classical linear beam theory (Eq.2). He showed that the ovalization of the cross-section of a long cylinder with increasing curvature induced by applied bending reduces its flexural rigidity. He then related the ovalization of the curved tube to the radial components of the tensile and compressive stresses in the wall-thickness. Using a nonlinear ring kinematic model, he proposed the following closed-form solution for the momentcurvature response of an infinitely long single tube.

$M_{B r}=\frac{2 \sqrt{2}}{9} \frac{E \pi r t^{2}}{\sqrt{1-v^{2}}} \approx 0.546 M_{c l}$

and a critical mean cross-sectional curvature

$$
\kappa_{\mathrm{Br}}=\frac{t \sqrt{2}}{3 r^{2} \sqrt{1-v^{2}}}
$$

Other researchers examined Brazier's moment theory [16, 19, 21, 25, 26, 36], and found that the Brazier moment is unachievable because premature bifurcation buckling occurs at a moment which is almost 5\% below $M_{B r}$ (Eq. 3). Comparison between Eqs. 2 and 3 suggests that the critical buckling moment of an infinitely long tube is about half of the critical buckling moment of the short tube. The effect of length on the buckling moment of tubes under bending action has been studied by many researchers $[16,18,19,21,36]$. One of the significant studies conducted by Rotter et al. [19] investigated the bending response of cylindrical shells with $D / t$ 
$\geq 40(r / t \geq 20)$ under geometrically nonlinear but elastic analyses. They used dimensionless geometric groups of $\omega$ and $\Omega$ as follows:

$$
\omega=\frac{L}{\sqrt{r t}}=L \sqrt{\frac{2}{D t}} \quad, \quad \Omega=\frac{L}{r} \sqrt{\frac{t}{r}}=\frac{2 L}{D} \sqrt{\frac{2 t}{D}}=\frac{t}{r} \omega
$$

and defined four distinctive length domains for the buckling behavior of tubes under bending; namely, short, medium, transitional and long cylinder, as represented in Table 1 . Their findings will be discussed in details later in this manuscript.

In civil and mechanical engineering applications, tubular shell structures such as silos and tanks may have radius-to-thickness ratios $(r / t)$ as large as 1000. However, in offshore pipes where the collapse under external pressure and burst due to the internal pressure [27] are the main design criteria, they usually inhabit a $D / t$ range of between $15-40(7.5<r / t<20)$. Kyriakides and Corona [28] investigated bending response of long pipes with length to diameter ratio $L / D$ $>20$ and $19.5 \leq D / t \leq 60.5$ using experimental, numerical and analytical studies. They found that in thick pipelines $D / t \leq 25$ failure occurs at moments slightly larger than the full plastic capacity $M_{p}$. The full plastic moment capacity of a tube is given as

$$
M_{P}=\sigma_{y} D^{2} t
$$

Sadowski and Rotter [29] investigated the bending behavior of thick shells which fail by plastic buckling well into the strain-hardening range using FE analysis. They illustrated how a relatively thick shell subjected to pure bending with characterized uniaxial tensile behavior of a mild steel when $D / t \approx 20(r / t \approx 10)$ may experience a limit point buckling due to combined extensive plasticity and ovalization. They observed no axial wrinkling in this thick shell. For a similar tube with $D / t \approx 50$, however, they showed that the shell undertakes considerable strainhardening and cross-sectional ovalization up to a limit point. They observed bifurcation 
buckling close to the peak moment accompanied by local axial wrinkling in post-buckling path [29].

All aforementioned studies were performed on single tubes and, to the knowledge of the authors, the bending response of double cylindrical tubes have been addressed only marginally so far [30-31]. In this paper, the behaviour of subsea PIP systems under the action of pure bending is investigated experimentally and numerically. PIPs with parameters represented in

Table 2 were selected covering the range of practical offshore applications. As real-life example of a PIP system, the Penguins pipeline in the Northern North Sea is a PIP system with a thick inner pipe $\left(D_{i} / t_{i}=19\right)$ and a thinner outer pipe $\left(D_{o} / t_{o}=35.15\right)$ [32]. Therefore, PIP-1 is considered to have a thin outer pipe and thick inner tube. To cover different PIP scenarios another PIP is defined (PIP-2) so that it has the opposite configuration and lastly PIP-3 has thick outer and inner tubes (ultra-deep subsea PIP system). Finite element analyses (FEA) are validated against published experimental results of single tubes in section 2 and are used to perform linear bifurcation analyse (LBA) and geometrically nonlinear analyses (GNA) presented in sections 3 and 4 respectively. The effect of length of PIPs on limit moments obtained from LBA and GNA analyses is discussed and, where possible, analytical expressions are proposed. The plastic bending moment and corresponding moment-curvature responses of PIPs are investigated experimentally and using geometrically and materially nonlinear analyse (GMNA) and the results presented in section 5. The paper concludes with a FEA parametric study on the effect of geometric and material parameters of the PIP system on its ultimate moment capacity.

\section{The finite element model and validation}

A schematic view of a PIP system is shown in Fig. 1a together with the boundary conditions and the adopted Cartesian coordinate system. Due to symmetry, only half cross-section of the 
PIP system with full length $L$ modelled with the commercial finite element software ANSYS [33]. In a typical PIP system, bulkheads are placed at both ends or several intervals in the pipeline to maintain the structural integrity of the system during installation and operation. As shown in Fig. 1a, bulkheads are welded to both the inner and outer pipes. In order to model the bulkheads in FEA at either ends of the PIP system ( $x=0$ and $x=L)$, the nodes on the inner and outer tubes (slave nodes) are coupled to the centroid of the cross-section (the master node) using MPC184 rigid link elements [33]. Equal moments of magnitude $M$ were simultaneously imposed onto the master nodes as shown in Fig. 1a. Due to existence of the rigid links, the PIP system is restrained from ovalization at the ends. Simply supported boundary conditions are imposed on the PIP system, by releasing the rotation $\left(\theta_{x}\right)$ of the master nodes about $x$ axis and restraining rotations about $y$ and $z$ axes. All translational degrees of freedom of the master node at $x=0$ are constrained, but the translation in $z$ direction of the master node at $x=L$ is released. Sadowski and Rotter [29] found that a thick 4 noded shell element with 6 degrees of freedom at each node would reasonably produce accurate estimations of the elastic-plastic strain hardening buckling moment under uniform bending for tubes as thick as $D / t$ ratio of $20(r / t=$ 10). Therefore, the outer and inner pipes are modelled using four-nodded Shell181 elements [33], with 6 degrees of freedom at each node. To obtain accurate results and to overcome convergence issues, 9 integration points in the pipe wall-thickness are designated. Based on the works of Rotter, Sadowski [29] a square mesh with 10 elements per local bending halfwave length $\lambda \approx 2.44 \sqrt{r t}$ in the circumferential and longitudinal directions was adopted for each tube.

The experimental results on bending of a single aluminium tube [34] were used to validate the current FE model. In the experimental tests conducted by Kyriakides and Ju [34], an aluminium pipe with $D=30.12 \mathrm{~mm}, t=1.63 \mathrm{~mm},(D / t=19.5), E=68.7 \mathrm{GPa}$ and yield stress $\sigma_{\mathrm{y}}=309$ MPa was used. In the current FEA, a bilinear isotropic material definition with $E^{\prime}=0.01 E$ is 
used ( $E^{\prime}$ is post-yield linear strain hardening modulus) and results are shown in Fig. 1b. In this figure, the moment is normalized to the plastic moment $M_{p}$ (Eq. 6) and curvature, $\kappa=2 \theta_{x} / L$, is normalised by the critical curvature $k_{\mathrm{c}}$

$$
\kappa_{c}=\frac{t}{D^{2}}
$$

Since the imperfection amplitude is not reported in the original study by Kyriakides and Ju [34], an imperfection sensitivity analysis is conducted herein. For this purpose an initial mesh perturbation in the form of the first corresponding eigen buckling mode shape was imposed on the model using Eigen buckling analyses. This produces a localised wrinkle imperfection $\bar{\omega}$ similar to axisymmetric initial imperfection given by

$$
\bar{\omega}=-\frac{D}{2}\left[a_{0}+a_{i} \cos \left(\frac{\pi x}{N \lambda^{\prime}}\right)\right] \cos \left(\frac{\pi x}{\lambda^{\prime}}\right)
$$

where $\lambda^{\prime}$ is the imperfection half-wave length and equal to $0.165 D, N$ is the number of halfwaves and equal to $11, a_{0}$ is the base amplitude and $a_{i}$ is biasing imperfection. In these analyses various base amplitudes of $2.50 \%, 1.25 \%$ and $0.25 \%$ corresponding to $0.03 D, 0.015 D$ and $0.003 D$ were imposed on the models and results are presented in Fig. 1b. Results shows that the FE model with the base amplitude of $0.003 D\left(a_{0}=0.25 \%\right)$ is in good agreement with the experimental results of Kyriakides and Ju [34]. The deformed shape of the FE model with imperfection of $0.003 D$ (shown in Fig. 1b) is similar to the experimental deformed shape. The negligible discrepancies at the yield curvatures, are known to be related to the inherent anisotropic behaviour of the aluminium and steel tubes in the hoop and longitudinal directions, due to the manufacturing process [35]. Moreover, as aluminium is known to be inherently nonlinear material, use of bilinear law to model the tube in FE is another source of error in the results. 


\section{Linear bifurcation analysis of PIPs (LBA)}

\subsection{Classical buckling moment of PIPs}

In the absence of imperfections or dents in the pipe wall, a tube under increasing curvature may develop buckles in the form of local short-wave wrinkling on the compression side, theoretically at compressive axial stresses equal to the critical stress $\sigma_{c l}$ defined in Eq. 1 . These wrinkles have opposite curvatures in both longitudinal and hoop directions. This type of instability, also known as bifurcation buckling, will typically occur in thin-walled tubes with large $D / t[20,29]$. Assuming a linear cross sectional stress distribution in the PIP system, it can be predicted that bifurcation buckling in PIPs will take place when the longitudinal compressive stress on either the inner or outer pipe reaches the classical buckling stress $\sigma_{c l}$ (Eq. 1). Thus, assuming that $\sigma$ is the stress in the mid-surface of the shell, the following critical stress conditions for bifurcation buckling of a PIP system can be obtained

$$
\begin{array}{ll}
\sigma_{c r-o}<\frac{D_{o}}{D_{i}} \sigma_{c r-i} & \text { Outer pipe buckles } \\
\sigma_{c r-o}>\frac{D_{o}}{D_{i}} \sigma_{c r-i} & \text { Inner pipe buckles }
\end{array}
$$

where subscripts $o$ and $i$, correspond to the outer and inner pipe, respectively. By substituting Eq. 1, into Eq.s 9a \& 9b and assuming the same material properties ( $E$ and $v$ ) in the outer and inner tubes, the following geometric conditions for bifurcation buckling of PIP system can be obtained

$$
\begin{array}{ll}
\frac{D_{i}}{D_{o}}<\frac{D_{o} / t_{o}}{D_{i} / t_{i}} & \text { The outer pipe buckles } \\
\frac{D_{i}}{D_{o}}>\frac{D_{o} / t_{o}}{D_{i} / t_{i}} & \text { The inner pipe buckles }
\end{array}
$$


Knowing that the contribution of moments in the outer and inner tubes is linearly proportional to their second moment of area, the following classical elastic buckling moments for bifurcation buckling of PIP system are derived as follow:

$$
M_{c l-P I P}= \begin{cases}\left(1+\frac{I_{i}}{I_{o}}\right) M_{c l-o} & \text { if } \frac{D_{i}}{D_{o}}<\frac{D_{o} / t_{o}}{D_{i} / t_{i}} \quad \text { The outer pipe buckles } \\ \left(1+\frac{I_{o}}{I_{i}}\right) M_{c l-i} & \text { if } \frac{D_{i}}{D_{o}}>\frac{D_{o} / t_{o}}{D_{i} / t_{i}} \quad \text { The inner pipe buckles }\end{cases}
$$

\subsection{Effect of length on linear critical buckling moment of PIPs (LBA)}

Previous studies $[16,19]$ have shown that the linear elastic buckling moments and shape and number of the wrinkles at the onset of buckling of the tubes, are significantly influenced by their lengths. Using Eigen-buckling analysis in ANSYS [33], and assuming isotropic linear material definition with parameters shown in Table $2\left(E_{i}=E_{o}=67 G P a\right)$, the elastic buckling moments of PIPs 1-3 with various lengths are calculated and presented in Figs. 2a-2c, respectively. The critical LBA moments are normalized by the classical buckling moment of PIPs proposed in Eq. 11. The abscissa is the non-dimensional length group, $\omega_{o}$ and $\Omega_{o}$ of the outer pipe of the PIP system defined in Eq. 5. According to Eq. 10, in all the long studied PIPs the buckling occurs in the outer pipe. Therefore, the length groups $\omega$ and $\Omega$ of the outer pipe are introduced herein. The linear meridional bending half-wavelength of the inner and outer tubes are calculated from $\lambda=2.44 \sqrt{r t}$ [37], and are displayed with dashed and solid vertical dividers in Fig. 2, respectively. Results are plotted up to $\Omega_{o}=0.5$ and, correspond to short and medium cylinders outlined in Table 1 . The buckling mode shapes of PIP1-3 and corresponding normalised lengths $\omega_{o}$, are depicted in Fig. 3, and show the formation of buckling in the outer or in the inner tube of the PIP system.

In very short PIP, where the length of the system is of the order of the bending half wave length of the outer pipe, the critical buckling moment is significantly higher than the classical buckling 
moment of the system proposed in Eq. 11. A similar trend is known to exist in the bifurcation buckling of very short single cylinders and, is related to the effect of boundary conditions on development of membrane stresses [19]. In the vicinity of boundaries, larger moments are required to cause a finite segment of the shell to reach its critical membrane stress [16, 21]. However, as the length of the system increases, the critical buckling moments converge towards the classical buckling moments of the PIPs (Eq. 11). This can be predicted from geometric dependency of elastic buckling outlined in by Eq. 10 .

With regards to Figs 2 and 3 it can be understood that in PIP-1, in which the outer pipe is thinner than the inner pipe, buckling occurs in the outer pipe regardless of the length. In PIP2, in which the outer pipe is thicker than the inner pipe, buckling occurs in the inner pipe when the length of the PIP system is smaller than bending half wave length of the outer pipe $\left(\lambda_{o}\right)$. At lengths longer than the bending half wave length of the outer pipe, buckling moves into the outer pipe. In PIP-3, with relatively thick inner and outer pipes, the buckling is confined within the inner tube when the length of the PIP is shorter than the bending half wave length of the inner pipe $\left(\lambda_{i}\right)$ and, moves to the outer pipe as the length of PIP increases. Results of linear bifurcation analyses (LBA) suggest that in PIPs within the transitional-to-long class $\left(\Omega_{0}>0.5\right)$ defined in Table 1, the classical elastic buckling moment $M_{c l}$ proposed in Eq. 11, can correctly predict bifurcation buckling of the PIP system.

\section{Geometrically Nonlinear Analysis of PIPs (GNA)}

\subsection{Limit moment of PIPs (Energy approach assuming plane-strain condition)}

Previous studies have shown that in single cylinders with $\Omega>0.5$ under bending, cross sectional ovalization occurs due to the formation of radial components of the tensile and compressive stresses [24]. With the increase of cross sectional curvature, ovalization is amplified and the lever arm between longitudinal components of tensile and compressive 
stresses is reduced. Subsequently, the flexural rigidity of the system is reduced and instability is observed at a moment much smaller than classical elastic buckling moment $M_{c l}$. The moment at which the section collapses due to ovalization is known as the Brazier moment.

Brazier [24] used an energy approach to predict the elastic nonlinear buckling moment of an infinitely long tube due to ovaliazation (Fig. 4a). Using small deflection theory and plane strain conditions, the condition that the radial displacement $(w)$ and tangential displacement $(v)$ are inextensional is that

$$
w=\frac{d v}{d \theta} \quad \text { OR } \quad \frac{d^{2} w}{d \theta^{2}}-\frac{d^{3} v}{d \theta^{3}}=-\kappa v r^{2} \cos \theta
$$

By neglecting the shear terms, Brazier [24] derived the following expression for the total strain energy per unit length of a bent single tube with cross sectional curvature $\kappa$

$$
U=\frac{E}{2} \pi \kappa^{2} r^{3} t\left(1-\frac{3}{4} \frac{r^{4} \kappa^{2}\left(1-v^{2}\right)}{t^{2}}\right)
$$

It has been seen in the FE analyses that the curvature of the inner and outer pipes are identical up to the appearance of wrinkles. Thus, adopting a similar approach and assuming that the radial and tangential displacements in the outer and inner tubes are in-extensional, the curvatures of the inner and outer pipes of the bent PIP are identical and equal to $\kappa$, then the total strain energy per unit length of a PIP system is

$$
U_{P I P}=\frac{E}{2} \pi \kappa^{2}\left[r_{o}^{3} t_{o}\left(1-\frac{3}{4} \frac{r_{o}^{4} \kappa^{2}\left(1-v^{2}\right)}{t_{o}^{2}}\right)+r_{i}^{3} t_{i}\left(1-\frac{3}{4} \frac{r_{i}^{4} \kappa^{2}\left(1-v^{2}\right)}{t_{i}^{2}}\right)\right]
$$

The moment curvature relationship becomes

$$
M=\frac{d U_{P I P}}{d \kappa}=E \pi \kappa\left[\left(r_{o}^{3} t_{o}+r_{i}^{3} t_{i}\right)-\frac{3}{2} \kappa^{2}\left(1-v^{2}\right)\left(\frac{r_{o}^{7}}{t_{o}}+\frac{r_{i}^{7}}{t_{i}}\right)\right]
$$


The limit curvature and the corresponding limit moment of the PIP system are calculated at the maximum $\left(\frac{d M}{d \kappa}=0\right)$. Limit curvature and limit moments of a single pipe can be calculated from Eqs (15a and 15b), if the radius of the inner pipe $\left(r_{i}\right)$ is reduced to zero.

$$
\begin{aligned}
& \kappa_{B r-P I P}=\sqrt{\frac{2}{9\left(1-v^{2}\right)} \frac{r_{o}^{3} t_{o}^{2} t_{i}+r_{i}^{3} t_{i}^{2} t_{0}}{r_{o}^{7} t_{i}+r_{i}^{7} t_{o}}} \\
& M_{B r-P I P}=\frac{2}{3} E \pi \kappa_{B r-P I P}\left(r_{o}^{3} t_{o}+r_{i}^{3} t_{i}\right)
\end{aligned}
$$

\subsection{Limit moment of PIPs (3D nonlinear geometric FEA)}

The influence of length on buckling moment of single cylinders is well-understood [16, 18, 21]. To account for the effect of length and possible non-uniform ovalization of the crosssection in the limit moment and limit curvatures of the PIP system, FE models of PIPs 1-3 are created. Isotropic linear material definition with parameters shown in Table 2 are adopted and geometrically nonlinear FE analysis (GNA) were performed, which account for geometric nonlinearities including large rotations, large deformations and stress stiffening, by forcing the solver to perform an iterative solution with an updated stiffness matrix according to the incremental nodal displacements at each equilibrium iteration [33]. To control the nonlinear analysis, an initial mesh perturbation in the form of the corresponding first Eigen-mode shape with maximum amplitude of $\delta_{o}=0.003 D$ is imposed on all the PIPs. Figure $4 \mathrm{~b}$ illustrates an enlarged view of the initial imperfection imposed on the outer pipe of the PIP system.

Normalised critical (LBA) and limit (GNA) moments of PIPs 1-3 are plotted against dimensionless length factors $\omega_{o}$ and $\Omega_{o}$, in Figs. 5a-5c, respectively. The elastic nonlinear moments derived in Eq. 16b are shown with the solid lines. As shown in Fig. 5, in short PIPs $\left(\Omega_{0}<0.5\right)$ the GNA moment is larger than the predicted elastic nonlinear moment. This is because in very short PIP systems a circumferential fold appears on the compression side of 
the outer or inner pipe (whichever buckles first). This fold becomes progressively deeper and the tangent stiffness increases as the analysis progresses. However, as the length of the PIP system grows the limit moment drops and converges to a constant moment at the plateaus in Fig. 5. In PIP-3 with relatively thick inner and outer pipes, the plateau in the GNA response is observed at $\Omega>2$, whereas in PIPs- 1 and 2 with thin outer and inner pipes, respectively, the plateau is observed in significantly longer PIPs. The GNA moment of the long PIPs 1-3 are 48.3\%, 51.5\% and 48.62\% lower than the LBA moment, respectively.

The GNA, classical (Eq. 11) and Brazier (Eq. 16b) moments of PIPs are represented in Table 3. While the Brazier moments of PIPs (Eq. 16b) slightly overestimate (3-8\%) the GNA, they are both about half of the classical moment (Eq.11). The difference between the Brazier moment of PIP and GNA result is because of occurrence of local buckling on the flatted compressed side which imposes a limit point failure through ovalization, consistent with single tubes $[14,36]$. Moreover, the accountability of Brazier moment formulation for PIPs is affected by the limitations of the formulation including assumption of plane strains and uniform ovalization across the length of the PIP system. While for cylinders with $\Omega<5$ maximum ovalization occurs in the midspan, in fact for tubes longer than $\Omega \approx 5$ the value of the ovalization at the midspan drops and the location of the maximum ovalization travels away from the midspan to the sides. This would result in a longer zone of full ovalization and consequently increases the bending resistance slightly [36]. Distribution of ovalization along the length of PIPs 1-3 with $\Omega_{0}=6.7$ (in the plateau regions of responses shown in Fig. 5), are plotted for the inner and outer pipes in Fig. 6. The ovalization $f_{0}$ (see Fig. 4a) at the limit curvature (Eq. 16a) of the inner or outer tube of each PIP is calculated from

$$
f_{0}=\frac{D_{\max }-D_{\min }}{D}=2\left[\frac{r^{4} \kappa^{2}}{t^{2}}\left(1-v^{2}\right)+\kappa v r\right]
$$


and is shown with solid and dashed-lines in Fig. 6. The graphs clearly indicate that in PIPs with $\Omega_{o}=6.7$ the maximum ovalization occurs away from the midspan and therefore ovalization along the length of inner and outer pipes are not uniform along the length. In PIPs 1 and 2 the maximum ovalization is observed almost at a quarter length away from the ends. Similar observation in single tube has been reported by other researchers [17, 34]. The constant Brazier ovalization calculated from Eq. 17 fail to predict the non-uniform ovalization patterns of the PIPs.

The normalised moment-curvature response of PIPs from the geometrically nonlinear finite element analyses (GNA) and the proposed Brazier expression for PIPs given in Eq. 15 are compared in Fig. 7. The proposed expression, Eq. 15 seems to predict the moment curvature behaviour of PIPs correctly. This is more apparent in PIP-3, where both outer and inner pipes are relatively thick. In all PIPs, the limit curvature obtained from Eq. 16a, is smaller than that of the GNA.

\subsection{Limit moment of PIPs with centralizers (3D nonlinear geometric FEA)}

The effect of the centralizers on the limit moment of PIPs 1-3 was investigated. To do so, in the FE model, two centralizers were mounted onto the inner pipes of PIPs 1-3. To investigate the possible interaction between the centralizers and the outer tube, the centralizers were positioned at locations with the maximum ovalization (see Fig. 6). To understand the effect of the centralizer's clearance, the length of the centralizers are kept constant $(25 \mathrm{~mm})$ while the height varies. The centralizer's clearance is defined as the gap between centralizers and the outer pipe, divided by the gap between inner and outer pipes (annulus). Mechanical properties of the centralizers were taken as $E=2.7 \mathrm{GPa}, \sigma_{y}=90 \mathrm{MPa}$ [38]. The centralizers were meshed using solid 185 elements, with 3 elements along the wall-thickness of the centralizer. 
Table 4 represents the normalised limit moments and limit curvatures of PIPs 1-3, with centralizer clearances of $12.5 \%$ and $25 \%$. For sake of comparison, the results of PIPs without centralizers are also presented. In all PIPs, the normalised limit moments get smaller as the centralizer clearances get larger. The limit curvatures of the PIPs with centralizers are smaller than those without centralizers. Analysis of deformed shapes (not shown here) reveals that, similar to PIPs with no centralizers, in PIPs with centralizers, the maximum ovalizations occur closer to the ends of the PIPs. However, upon the contact between the outer pipe and the centralizers, the maximum ovalization moves towards the mid-span of the outer pipe. From this point ovalization continues to grow in the middle of the system, until the limit moment is reached.

\section{Ultimate bending capacity of PIPs (GMNA)}

\subsection{Geometric and Material Nonlinear Analysis of PIPs (GMNA)}

Previous studies on the ultimate moment capacity of offshore pipelines with $15<D / t<40$ (7.5 $<r / t<20$ ) have shown that the failure mode in bending is dominated by the material plasticity $[14,19,25]$. Wang et al. [21] found that, in contrast to the thin cylindrical shells, ovalization of thick cylinders due to bending is only responsible for a very minor reduction (less than 5\%) in the full plastic moment capacity. In order to investigate the effects of material plasticity on the bending response of PIPs, materially nonlinear but geometrically linear analysis (MNA) and geometrically and materially nonlinear analysis (GMNA) were performed on PIP-1, 2 and 3. A bilinear isotropic material definition with the stress-strain relation shown in Fig. 9 was assumed. The inner and outer pipes were assumed to have similar material properties, $E_{i}=$ $E_{o}=67 G P a, E_{i}^{\prime}=E_{o}^{\prime}=670 M P a$ and $v_{i}=v_{o}=0.3, \sigma_{y o}=\sigma_{y i}=170 \mathrm{MPa}$. The MNA and GMNA results are shown in Fig. 7, where the moments are normalised to the plastic moment of the PIP system 


$$
M_{P-P I P}=\sigma_{y o} D_{o}^{2} t_{o}+\sigma_{y i} D_{i}^{2} t_{i}
$$

The GMNA moments of PIPs $1-3$ are $20 \%, 12 \%$ and $10 \%$ of the corresponding GNA moments, respectively. In PIP-1, with the thinnest outer pipe, the largest $M_{G M N A} / M_{c l-P I P}$ ratio is observed which is almost twice the ratio in PIPs 2 and 3. It is shown that similar to thick single tubes [21] there is a negligible difference between results of GMNA and MNA analyses of PIPs in bending. All PIPs fail by plastic collapse with a very limited influence of cross sectional ovalization at a moment which is close to the plastic moment capacity of the system (Eq. 18). In all PIPs, the GMNA follows the MNA path up to a certain plastic curvature. In PIPs 1 and 2, a clear drop in the capacity following the ultimate GMNA moment is observed. The drop is more pronounced in PIP-1 with thin outer pipe. However, in PIP3, (with relatively thick outer and inner pipes), at the ultimate GMNA moment the implicit FE solver fails to converge. This is mainly due to the reason that PIP3 with $D / t$ ratio of 20 is at the limit of what can be modelled using a thick shell treatment [29]. It is worth noting that as it is expected, in all PIPs the curvature at the ultimate GMNA is also smaller than the corresponding limit curvature (GNA).

\subsection{Effect of centralizers on the GMNA of PIPs 1-3}

The effect of centralizer on the bending responses of PIPs 1-3 was investigated using FE with geometric and material nonlinearities. One centralizer with properties defined in section 4.3 was mounted onto the inner pipe and at the mid-length of the PIP system (where the maximum deformation at the ultimate moment of the PIPs without centralizers was observed). Schematic deformed shape of the PIP-1 with centralizer clearance of 5\% at normalised curvature of 1.5 is shown in Fig. 8a. The moment curvature response of PIPs 1-3 with difference centralizer clearances are shown in Fig. 8. It is evident that the ultimate moments of the PIPs are not affected by the addition of the centralizers. As shown in Fig. 8a, in PIP-1, the centralizers have a stabilizing effect on the post-buckling response of the system. In PIPs 2 and 3, the curvature 
at ultimate moment of the PIP system becomes smaller as the centralizer clearance gets larger. However, the curvature at the ultimate moment of PIP-1 seems to be unaffected by the centralizer clearance.

\subsection{Experimental investigations}

\subsubsection{Mechanical properties and the test set-up}

The bending response of PIP-1 was studied further using a 4-point bending test. Three identical PIPs are made up of aluminum (AL-6060-T5) inner and outer pipes with geometrical parameters represented in Table 5. The numerical study in section 5.2 of PIP-1 showed that addition of centralizers has a stabilizing effect on the post-buckling response of the system. Therefore, no centralizers were added to the PIP system in the experimental study of bending. Three coupon samples were water jet cut from the inner and outer tubes of each PIP sample according to the specifications of AS 1391 [39] and were tested in a unidirectional tensile test. The stress-strain curve of the outer pipe is shown in Fig. 9, and the mechanical properties of each pipe are represented in Table 4. The PIP samples were fabricated by inserting the inner pipe inside the outer pipe and holding them concentrically using a fast curing two parts Epoxy resin at both ends. Two acrylic diaphragms were placed on either ends of the PIP sample in order to confine the Epoxy fillings, as shown in 10a. All samples were cut into $550 \mathrm{~mm}$ long specimens leaving a $400 \mathrm{~mm}$ free span between the restrained Epoxied regions. Based on the results of the LBA and GNA of PIP-1 provided in Sections 3 and 4 respectively, the length of the PIP sample was taken as $400 \mathrm{~mm}$ which is equivalent to $\Omega_{o}=2.24$. This ensures that the PIP sample is long enough to avoid the boundary condition effects and the full limit curvature/moment can be developed. Considering that the ultimate moment of PIP-1 is not influenced by the centralizer (refer to the previous section), no centralizer was installed on the test samples. 
A schematic view of the four-point bending set-up is shown in Fig. 10a and the test rig showing the failed sample is depicted in Fig. 10b. The vertical load $(F)$ was applied by a hydraulic jack onto a spreader beam (a wide-flange steel beam with web-stiffeners) which rests on two steel rectangular hollow sections (RHS). To release rotations, a wedge and a roller shaft were used to equally transfer the load $F$ from the spreader beam into the steel RHS profiles. The PIP sample was mounted on two steel end-plates bolted to the steel RHS profiles. The assembly was simply supported at both ends, by steel RHS profiles on vertical posts using pinned joint and slotted-hole mechanisms. As shown in Fig. 10a, the test rig applies a constant moment of F/2×455mm to the PIP system.

The curvature of the PIP system during bending was measured using three linear variable differential transducers (LVDTs) located under the PIP and at mid-span and quarter-span. To monitor the ovaliztion of the samples, an ovalization recorder device was manufactured and mounted on the outer pipe of the PIP system. The ovalization recorder is shown in Fig. 11 and consists of 4 laser detectors placed on two fixed jaws and one mobile jaw using two $8 \mathrm{~mm}$ steel rods and two springs. The ovalization recorder can move freely with the sample as it undergoes curvature (Fig. 11). The top and bottom laser detectors measure the flattening of the crosssection $\left(D_{\min }\right)$, while the side laser detectors measure the horizontal extension $\left(D_{\max }\right)$. Knowing the maximum and minimum diameters, the ovaliztion in the outer tube $f_{0}$ may be calculated from Eq. 17. The ovalization recorder was also used to measure the initial imperfection in tubes prior to conducting the bending tests. To do so, the sample was placed into the assembly shown in Fig. 11 with only the two side laser detectors. The diameter of the pipe was measured in $45^{\circ}$ intervals by rotating the pipe inside the assembly and the ovality was calculated from Eq. 17. Using this method, the maximum recorded ovaliztion of tubes was 0.007 , which is well below the allowable ovalization of 0.03 recommended in DNV [40]. 


\subsubsection{Comparison between experimental and GMNA results}

The normalised moment-curvature response and the failed PIP-1 samples from three repeats are shown in Fig. 12. For sake of comparison the GMNA response from the FEA and its deformed shape are also depicted on the figure. As shown in the failed deformed shapes in PIP1, test \#1 and PIP-1, test \#3, the local buckling did not occur in the mid-length. However, in test \#2 the failure was closer to the middle of the sample. The GMNA moment-curvature response (Fig. 12a) and failed deformed shape (Fig. 12b) are in better agreement with test \#2. As expected in PIP-1 with thin outer pipe, the ultimate moments found from the experimental tests and the GMNA, are lower than the plastic moment (Eq. 18).

The moment-ovalization response of PIP-1, test \#2 and that of GMNA are plotted in Fig. 13. Only results of test\#2 are plotted, since the ovaliztion in that test was measured at the midlength were the local failure occurred. A good agreement between the moment-curvature response and moment-ovaliztion behaviour from the experiments and GMNA results are observed in Figs. 12 and 13.

\subsection{Effect of $D_{i} / D_{o}$ and $t_{i} / t_{o}$ on the ultimate moment and curvature capacity of PIPs using FEA (GMNA)}

To investigate the effect of $D_{i} / D_{o}$ and $t_{i} / t_{0}$ ratios on the ultimate moment and curvature capacities of the PIPs, two sets of PIPs with $D_{o} / t_{o}$ ratios of 26.7 and 40 were modelled using GMNA. The inner and outer pipes are assumed to be of the same material, $\sigma_{y, i}=\sigma_{y, o}=$ $170 \mathrm{MPa}, E_{i}=E_{o}=67 \mathrm{GPa}$ and ${E^{\prime}}_{i}={E^{\prime}}_{o}=670 \mathrm{MPa} . \mathrm{D}_{o} / t_{o}$ ratios were kept constant, and the diameter and thickness of the inner pipes were varied. The results are shown in Fig.14a and 14b for $D_{o} / t_{o}$ of 26.7 and 40 respectively. Equation 9 is shown as a plane on the figures and clearly identifies the onset of failure in the outer or the inner pipe. In PIP with thin outer pipe (Fig. 14b) the ultimate moments are slightly less than the plastic moment. At small $D_{i} / D_{o}$, an increase in $t_{i} / t_{0}$ appears to have no significant influence on the ultimate moment capacity of the system, whereas at small $t_{i} / t_{o}$ ratios the ultimate moment capacity drops with the increase 
of $D_{i} / D_{o}$. As shown in Fig. 14a, in PIPs with a thicker outer pipe, ultimate moments are slightly larger than the plastic moment. The ultimate moment capacity of the PIP system decreases with the corresponding increase in thickness ratios. Similar to the PIPs with thinner outer pipe, in PIPs with thicker outer pipe as $D_{i} / D_{o}$ ratio grows while $t_{i} / t_{o}<0.8$ ultimate moment capacity reduces. This is mainly due to the reason that the onset of failure occurs in the inner pipe. In PIP with thinner outer pipe $(D o / t o=40)$ ultimate curvature capacity is less than the critical curvature of the outer pipe in a single pipe system. The opposite is true for the PIPs with thicker outer pipe $(D o / t o=26.7)$. In both group of PIPs when $t_{i} / t_{o}>0.8$ increase of $D_{i}$ / $D_{o}$ ratio would marginally influence the ultimate curvature capacity of the system, whereas increase of $D_{i} / D_{o}$ in PIPs with $t_{i} / t_{o}<0.8$ results in the drop of ultimate curvature capacity significantly. Among all PIPs shown in Fig. 14 the ultimate moments and curvatures capacities are minimums when the failure occurs in the inner pipe.

\subsection{Effect of $\sigma_{y i} / \sigma_{y o}$ on the ultimate moment and curvature capacities of PIPs using FEA (GMNA)}

The API [41] provides specifications of the pipeline material appropriate for conveying oil, gas and water in oil and gas industry. API [41] recommends the use of different steel grades (X52 to X70) with yield strength ranging from 359 to $483 \mathrm{MPa}$ (52 to $740 \mathrm{ksi}$ ). For a typical PIP system the $\sigma_{y i} / \sigma_{y o}$ ratio might vary. For example, the Shell Penguins $60 \mathrm{~km}$ long PIP system is comprised of an inner pipe with $\sigma_{y i}=420 \mathrm{MPa}$ and an outer pipe with $\sigma_{y o}=450 \mathrm{MPa}$ [32]. Therefore, the effect of $\sigma_{y i} / \sigma_{y o}$ on the ultimate moment and curvature capacities was investigated in PIPs with outer and inner pipes of different diameter-to-thickness ratios. In all models, the yield stress of the inner pipe was alternated while the yield stress of the outer pipe was held constant. The results are presented in Figs.15a for PIPs with inner pipe almost as thick as the outer pipe $\left(t_{i} / t_{0}=1\right)$ and in Fig.15b for PIPs with outer pipe thinner than the inner pipe $\left(t_{i} / t_{0}<1\right)$. The largest variation in the normalised moment capacity (up to 15\%) is observed in 
PIPs with thin outer pipe (Figs.15a), whereas the largest change in the normalised ultimate curvature occurs in PIPs with thicker inner pipe (Figs.15b). In PIPs with thick outer pipes (Figs.15b), the ultimate moment is less affected by the corresponding change in the yield ratio, while ultimate curvature increases with the growth of $\sigma_{y i} / \sigma_{y o}$. Results show that in PIPs with thin outer pipe any growth in the $\sigma_{y i} / \sigma_{y o}$ ratios results in larger ultimate curvature up to $\sigma_{y i} / \sigma_{y o}=1$. After this point any increase of $\sigma_{y i} / \sigma_{y o}$ ratio reduces ultimate curvature of the system. As shown in the bottom of Fig.15b, in PIP with relatively thick inner and outer pipes and small annulus $\left(D_{i} / D_{o}=0.75\right)$, failure always occurs in the inner pipe and the ultimate moments are consistently close to the plastic moment capacity.

\section{Conclusion}

The flexural response and instability moments in a 'pipe-in-pipe' system (PIP) with $15<D / t<$ 40 (Table 2) were investigated experimentally and numerically. The classical buckling moment of the PIP system was calculated and the condition of its occurrence in the outer or inner tube was defined. The proposed expression was compared to results of the FE linear bifurcation analysis (LBA). It was shown that in long PIPs, the proposed algebraic expression (Eq. 11) almost matches the classical moment found from a linear bifurcation analysis. It was shown that, a geometric criterion can correctly predict the occurrence of the linear buckling failure in the outer or inner pipe. Using a strain energy approach with plane strain conditions, equations for limit moments and curvatures of PIPs (Eqs. 15a and 15b) were developed. These expressions were shown to be able to predict the GNA moment-curvature response of PIPs with good accuracy. It was shown that in long PIPs, the GNA and Brazier moments are 45\% to $50 \%$ lower than the corresponding classical (LBA) moments. The effect of centralizers on the limit moments of PIPs was studied by introducing centralizers at locations with maximum 
ovalization. It was understood that, compared to PIPs without centralizers, in PIPs with centralizers, both the limit moments and limit curvatures drop.

Ultimate bending moments of PIPs were obtained from physical four-point bending tests of PIP-1 and geometrically and materially nonlinear analyses (GMNA). The flexural response, ovalizations and ultimate moments predicted by GMNA were shown to be in good agreement with the experimental results. The results showed that for the investigated PIPs with thick tubes, ovalizations at the ultimate moment were almost negligible. Moreover, finite element GMNA studies of PIPs with centralizers showed that the ultimate moments are not affected by the existence of the centralizers. Results of the parametric study on ultimate moments of PIPs revealed that, similarly to single-walled pipelines, PIPs with thick outer tubes exhibit ultimate moments slightly larger than the full plastic moment (Eq. 18). It was understood that the ultimate moment capacity of the PIPs decreases with the corresponding increase in thickness ratios as long as $t_{i} / t_{0}<1$. Minimum ultimate moments were observed in PIPs at which the inner pipe failed. In PIPs with thick outer pipes, the ultimate moments were less affected by the corresponding change in the yield ratio of the outer to inner pipe. Whereas, in PIPs with a thin outer pipe, variation in the yield ratios showed up to $15 \%$ difference in the moment capacity of the PIP system. 


\section{References}

[1] P. Jukes, A. Eltaher, J. Sun, G. Harrison, Extra High-Pressure High-Temperature (XHPHT) Flowlines-Design Considerations and Challenges. in Proceedings of the ASME 28th International Conference on Ocean, Offshore and Arctic Engineering, (2009) 469-478.

[2] H. Karampour, F. Albermani, P. Major, Interaction between lateral buckling and propagation buckling in textured deep subsea pipelines, ASME 2015 34th International Conference on Ocean, Offshore and Arctic Engineering, 2015.

[3] H. Karampour, Effect of proximity of imperfections on buckle interaction in deep subsea pipelines, Marine Structures, 59 (2018) 444-457.

[4] Z. Wang, Z. Chen, H. Liu, Numerical study on upheaval buckling of pipe-in-pipe systems with full contact imperfections, Eng. Struct. 99 (2015) 264-271.

[5] M.A. Vaz, M.H. Patel, Lateral buckling of bundled pipe systems, Mar. Struct. 12 (1999) 21-40.

[6] H. Karampour, F. Albermani, J. Gross, On lateral and upheaval buckling of subsea pipelines, Eng. Struct. 52 (2013) 317-330.

[7] C. Langner, R. Ayers, The feasibility of laying pipelines in deep waters, in: Proceedings of the 4th Offshore Mechanics and Arctic Engineering Symposium, 1985.

[8] H. Karampour, F. Albermani, M. Veidt, Buckle interaction in deep subsea pipelines, Thin-Walled Struct. 72 (2013) 113-120.

[9] H. Karampour, M, Alrsai, F. Albermani, H. Guan, D. Jeng, Propagation Buckling in Subsea Pipein-Pipe Systems. Journal of Engineering Mechanics, 143 (2017) 04017113.

[10] M. Alrsai, H. Karampour, F. Albermani, On collapse of the inner pipe of a pipe-in-pipe system under external pressure. Engineering Structures, 172 (2018) 614-628.

[11] M. Alrsai, H. Karampour, F. Albermani, Numerical study and parametric analysis of the propagation buckling behaviour of subsea pipe-in-pipe systems. Thin-Walled Structures, 125 (2018) 119-128.

[12] P. Stephan, C. Love, F. Albermani, H. Karampour, Experimental study on confined buckle propagation, Advanced Steel Construction, 12 (2016) 44-54.

[13] S. Kyriakides, Buckle propagation in pipe-in-pipe systems.: Part I. Experiments, International Journal of Solids and Structures, 39 (2002) 351-366.

[14] S. Kyriakides, T.J. Vogler, Buckle propagation in pipe-in-pipe systems.: Part II. Analysis. International Journal of Solids and Structures, 39 (2002) 367-392.

[15] H. Karampour, F. Albermani, Buckle interaction in textured deep subsea pipelines, Ships Offshore Struct. 11 (2016) 625-635.

[16] O. Fajuyitan, A.J. Sadowski, Imperfection sensitivity in cylindrical shells under uniform bending. Advances in Structural Engineering, 21(16) (2018) 2433-2453.

[17] G. Ju, S. Kyriakides, Bifurcation and localization instabilities in cylindrical shells under bending-II. Predictions. International journal of solids and structures, 29 (1992) 1143-1171. 
[18] L. Li, R. Kettle, Nonlinear bending response and buckling of ring-stiffened cylindrical shells under pure bending. International Journal of Solids and Structures, 39 (2002) 765-781.

[19] J.M. Rotter, A.J. Sadowski, L. Chen, Nonlinear stability of thin elastic cylinders of different length under global bending. International Journal of Solids and Structures, 51 (2014) 2826-2839.

[20] P. Seide, V.I. Weingarten, On the buckling of circular cylindrical shells under pure bending. Journal of Applied Mechanics, 28 (1961) 112-116.

[21] J. Wang, A.J. Sadowski, J.M. Rotter, Influence of ovalization on the plastic collapse of thick cylindrical tubes under uniform bending. International Journal of Pressure Vessels and Piping, 168 (2018) 94-99.

[22] S.P. Timoshenko, S. Woinowsky-Krieger, Theory of plates and shells, McGraw-hill 1959.

[23] L.H. Donnell, Stability of thin-walled tubes under torsion, 1935.

[24] L. Brazier, On the flexure of thin cylindrical shells and other thin sections, Proceedings of the Royal Society of London. Series A, containing papers of a mathematical and physical character, 116 (1927) 104-114.

[25] B.F. Tatting, Z. Gürdal, V.V. Vasiliev, The brazier effect for finite length composite cylinders under bending. International Journal of Solids and Structures, 34 (1997) 1419-1440.

[26] S.A. Karamanos, Bending instabilities of elastic tubes, Solids and structures, 39 (2001) 20592085.

[27] S. Kyriakides, E. Corona, Mechanics of Offshore Pipelines. Elsevier, 2007.

[28] S. Kyriakides, A. Ok, E. Corona, Localization and propagation of curvature under pure bending in steel tubes with Lüders bands, International Journal of Solids and Structures, 45 (2008) 30743087.

[29] A.J. Sadowski, J.M. Rotter, Solid or shell finite elements to model thick cylindrical tubes and shells under global bending. International Journal of Mechanical Sciences, 74 (2013) 143-153.

[30] I. Nikiforov, D. Zhang, R. James, T. Dumitrică, Wavelike rippling in multiwalled carbon nanotubes under pure bending. 96 (2010).

[31] C. Jolicoeur, A. Cardou, Analytical Solution for Bending of Coaxial Orthotropic Cylinders, 120 (1994) 2556-2574.

[32] M. Carr, I. Matheson, R. Peek, P. Saunders, N. George, Load and Resistance Modelling of the Penguins Pipe-in-Pipe Flowline Under Lateral Buckling. ASME, (2004) 39-47.

[33] ANSYS 17.0 Release, Ansys Inc. 275 Technology Drive, Canonsburg, PA 15317.

[34] S. Kyriakides, G. Ju, Bifurcation and localization instabilities in cylindrical shells under bending-I. Experiments, International Journal of Solids and Structures, 29 (1992) 1117-1142.

[35] E. Corona, L.-H. Lee, S. Kyriakides, Yield anisotropy effects on buckling of circular tubes under bending. International Journal of Solids and Structures, 43 (2006) 7099-7118.

[36] Z. Xu, L. Gardner, A.J. Sadowski, Nonlinear stability of elastic elliptical cylindrical shells under uniform bending. International Journal of Mechanical Sciences, 128 (2017) 593-606. 
[37] A.J. Sadowski, L. Pototschnig, and P. Constantinou, The 'panel analysis' technique in the computational study of axisymmetric thin-walled shell systems. Finite Elements in Analysis and Design, 152 (2018) 55-68.

[38] Thermoplastic components for the Oil \& Gas industry, in Innovative Thermoplastic Engineering, J. Walker, Editor. 2018: United Kingdom.

[39] A. Standard, AS 1391. Metallic materials-Tensile testing at ambient temperature, Standards Australia, 2007.

[40] DNV, Recommended practice DNV-RP-F101 corroded pipelines. Hovik, Norway, (2004) 135138.

[41] API, 5L, Specification for Line Pipe. Edition March, 2004. 


\section{List of Figures}

Fig. 1. (a) The FE model and boundary conditions (b) comparison between experimental results of Kyriakides and Ju [1992] and FE results of aluminium pipe with $D / t=19.5$

Fig. 2. Influence of dimensionless length parameter on the linear elastic buckling moment of (a) PIP-1 $\lambda_{o}=21.82 \mathrm{~mm}, \lambda_{i}=13.80 \mathrm{~mm}$, (b) PIP-2 $\lambda_{o}=27.60 \mathrm{~mm}, \lambda_{i}=10.91 \mathrm{~mm}$ and (c) PIP$3 \lambda_{o}=30.86 \mathrm{~mm}, \lambda_{i}=17.25 \mathrm{~mm}$

Fig. 3. Linear elastic buckling modes for PIP-1, 2 and 3 with different lengths subjected to global bending

Fig. 4. (a) Assumed Geometry and coordinate system to describe the ovalization of a single tube (b) Enlarged mesh of the PIP system used in the GNA, showing the imposed mesh perturbation

Fig. 5. Influence of dimensionless length parameter on the linear (LBA) and nonlinear (GNA) elastic buckling moment of (a) PIP-1 (b) PIP-2 (c) PIP-3

Fig. 6. Ovalization along the length of (a) PIP-1 (b) PIP-2 (c) PIP-3 with $\Omega_{o}=6.7$ obtained from GNA

Fig. 7. Normalised moment vs. normalised curvature of GNA, MNA and GMNA of (a) PIP-1 (b) PIP-2 (c) PIP-3

Fig. 8. Effect of centralizers with different clearances on the nonlinear geometry and nonlinear material analyses (GMNA) of (a) PIP-1 (b) PIP-2 (c) PIP-3

Fig. 9. Stress-strain relationship of the pipe with $\mathrm{D}=80 \mathrm{~mm}$ and $\mathrm{t}=2 \mathrm{~mm}$ (outer pipe of PIP1 in Table 4) obtained from a unidirectional tensile test and the bi linear model adopted in the FEA

Fig 10. (a) Schematic view of the four-point bending test set-up (b) Image of four-point bending rig

Fig. 11. (a) Schematic view of the ovalization recorder used to measure ovality of the outer pipe during the bending test (b) Photo of the ovalization recorder in use

Fig. 12. (a) Experimental and FEA (GMNA) bending response of PIP-1 samples in pure bending, (b) the failure modes from experiments and FEA

Fig. 13. Normalised moment-ovalization response of the outer pipe of PIP-1 from experiment and GMNA

Fig. 14. Effect of $D_{i} / D_{o}$ and $t_{i} / t_{o}$ on ultimate moment and curvature capacities of (a) PIP with $D_{o} / t_{o}=26.7$ and, (b) PIP with $D_{o} / t_{o}=40$

Fig. 15. Effect of $\sigma_{y i} / \sigma_{y o}$ ratio on the ultimate moment capacity of PIP systems with (a) inner pipe almost as thick as the outer pipe and (b) outer pipe thinner than the inner pipe 


\section{List of Tables}

Table 1. Classification and characterisation of elastic isotropic cylindrical pipes in bending for $\mathrm{D} / \mathrm{t} \geq 40$ [19]

Table 2. Geometrical properties of the PIP systems used in LBA and GNA

Table 3. Comparison between the GNA, LBA and Brazier moments of long PIPs $(\Omega>7)$

Table 4. Effect of centralizers on limit moment and limit curvature of PIPs 1-3 (GNA)

Table 5. Properties of the PIP-1 used in the experimental study 


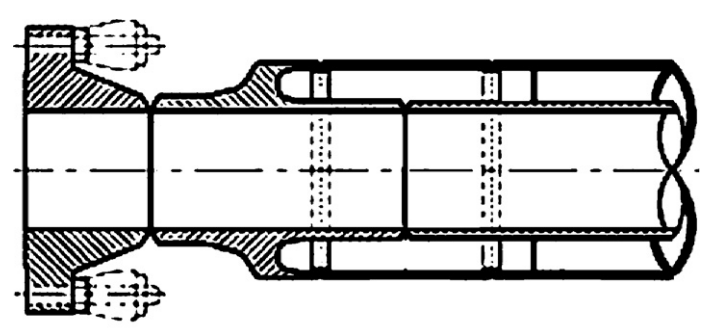

End bulkhead configuration

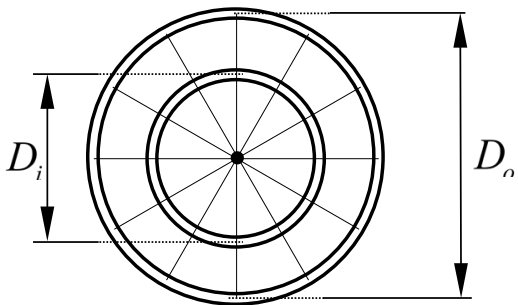

PIP's cross sectional view

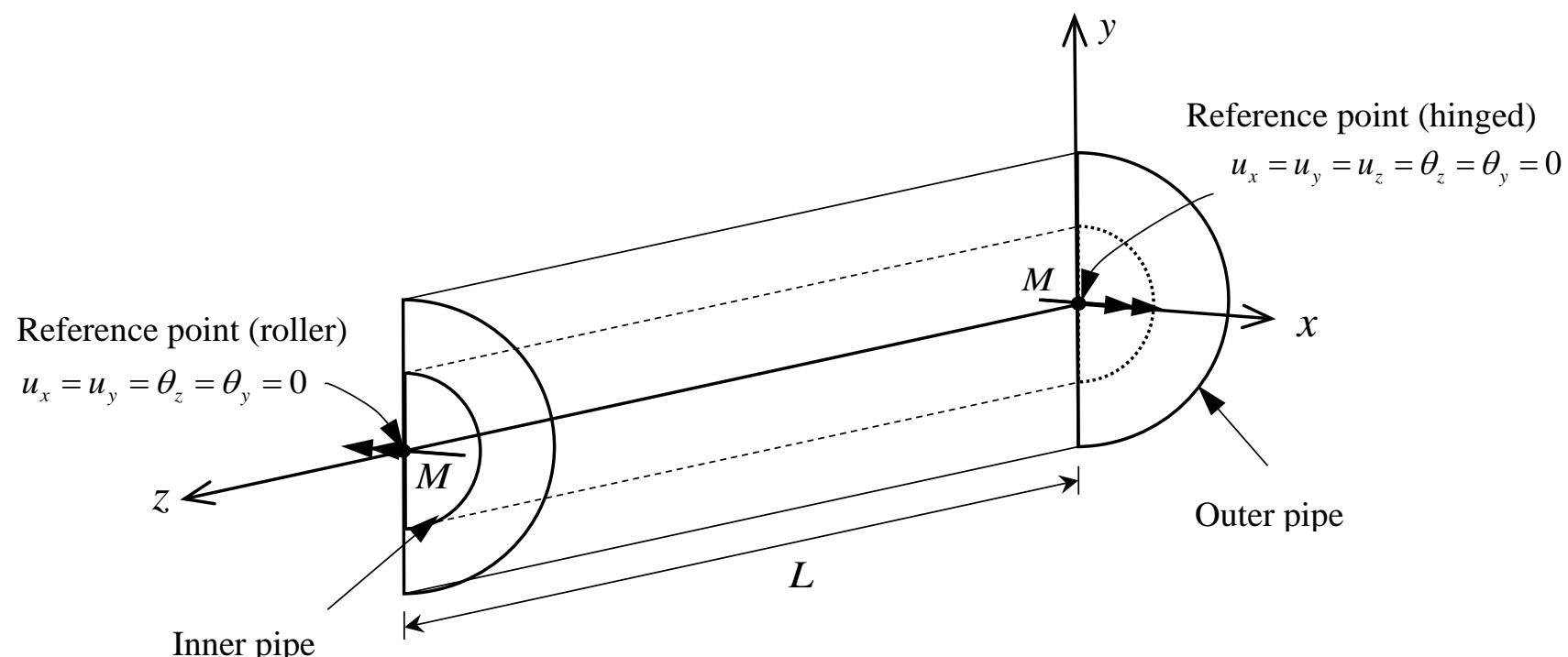

a

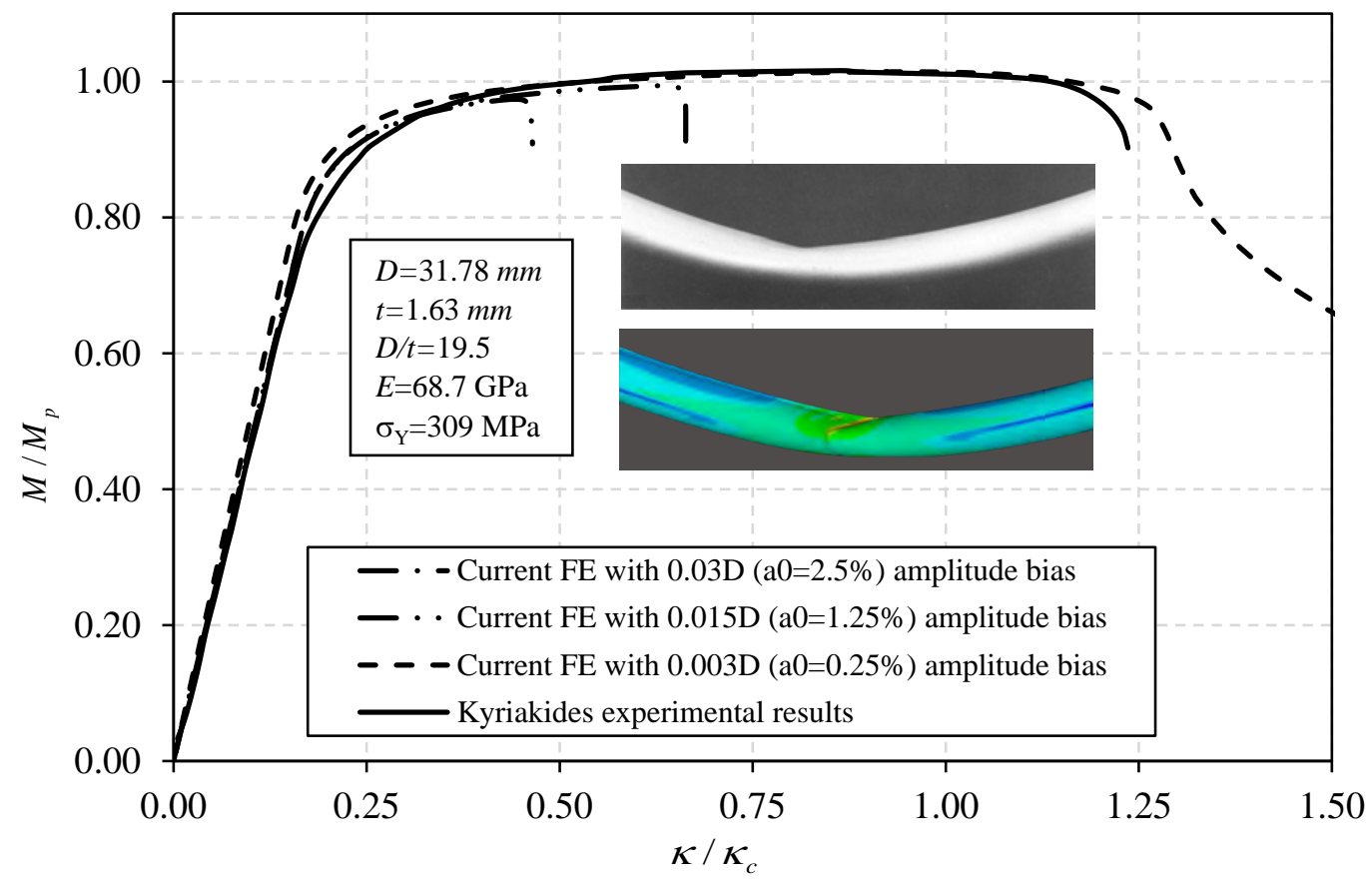

b

Figure 1 (a) The FE model and boundary conditions (b) comparison between experimental results of Kyriakides and Ju (1992) and FE results of aluminium pipe with $D / t=19.5$ 
a) $\quad \Omega_{o}=\frac{L}{r_{o}} \sqrt{\frac{t_{o}}{r_{o}}}=\frac{t_{o}}{r_{o}} \omega_{o}$

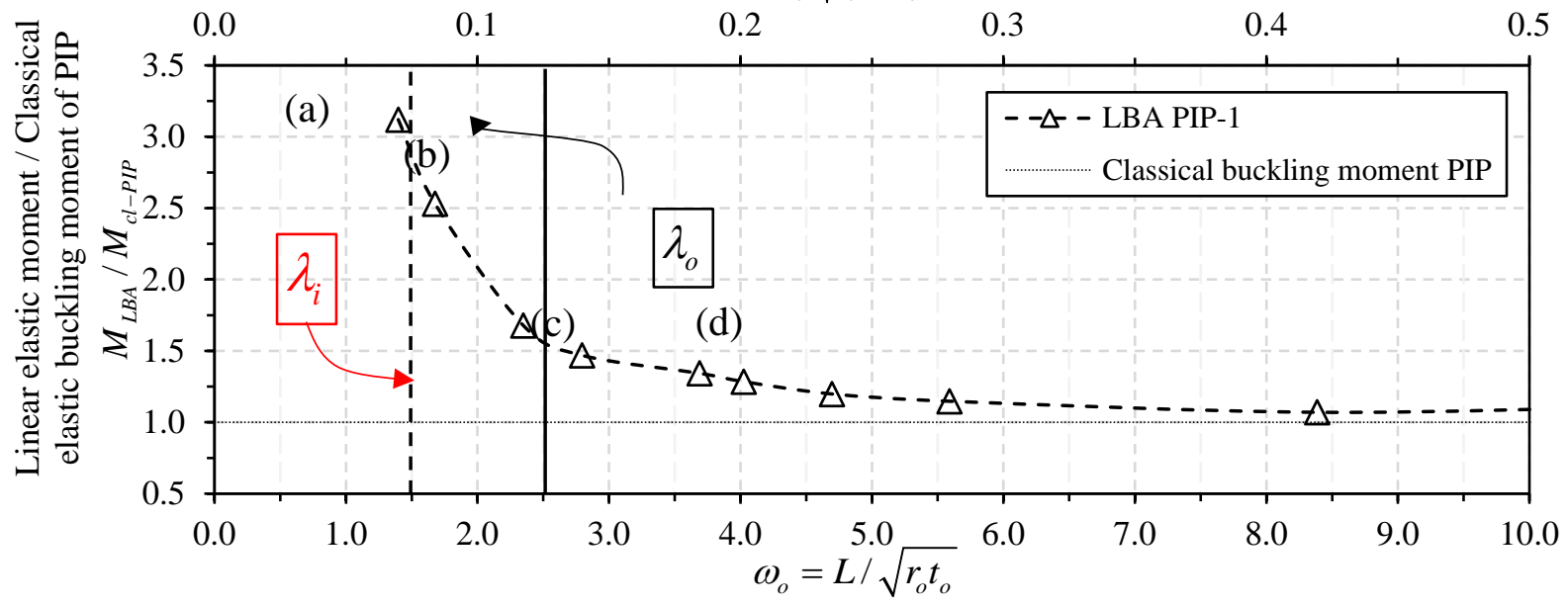

b)

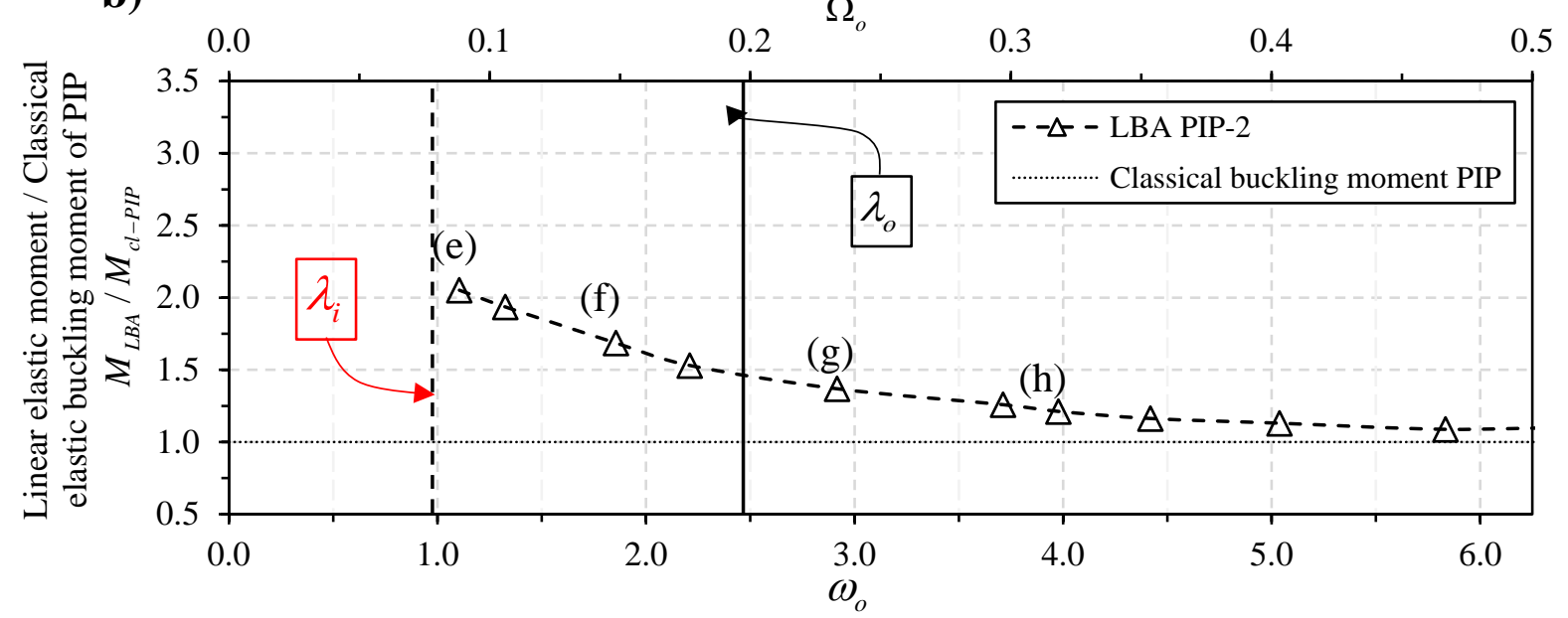

c)

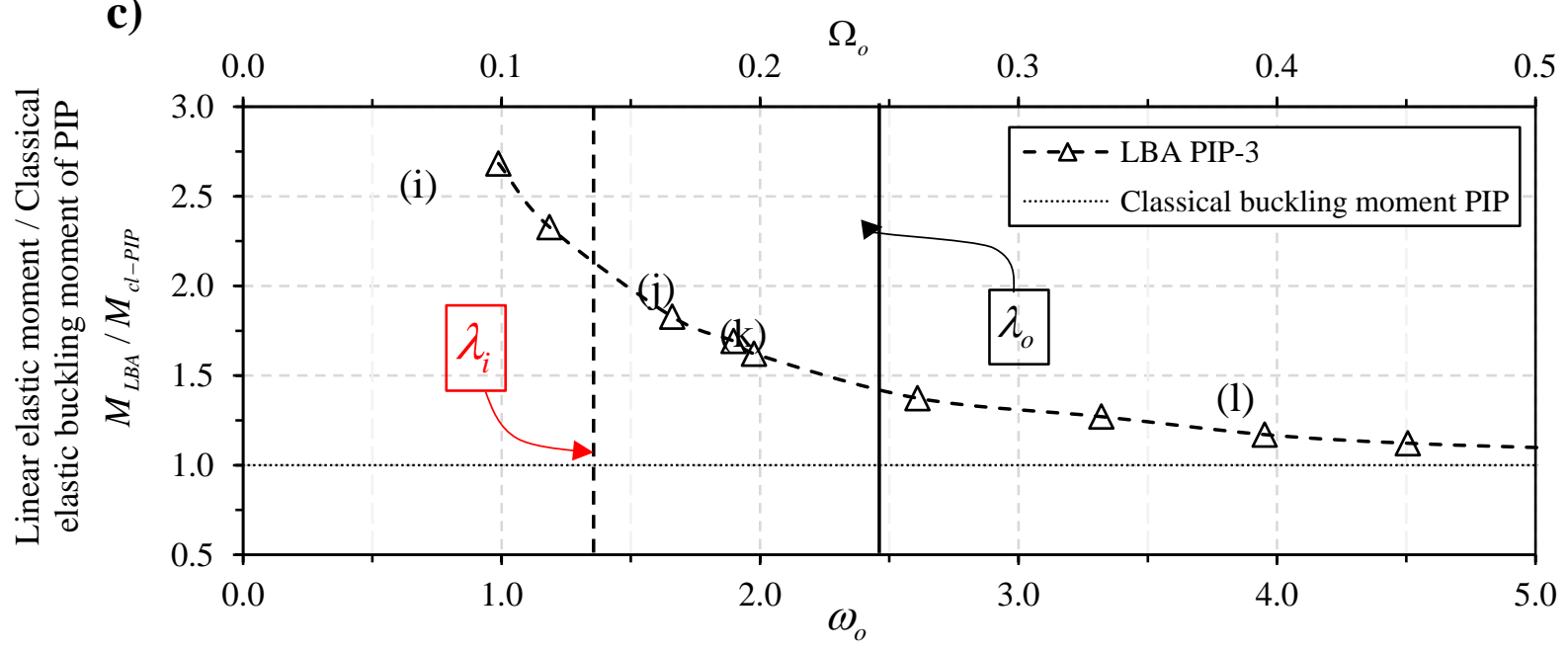

Figure 2 Influence of dimensionless length parameter on the linear elastic buckling moment of (a) PIP-1 $\lambda_{o}=21.82 \mathrm{~mm}, \lambda_{i}=13.80 \mathrm{~mm}$, (b) PIP-2 $\lambda_{o}=27.60 \mathrm{~mm}, \lambda_{i}=10.91 \mathrm{~mm}$ and (c) PIP-3

$$
\lambda_{o}=30.86 \mathrm{~mm}, \lambda_{i}=17.25 \mathrm{~mm}
$$




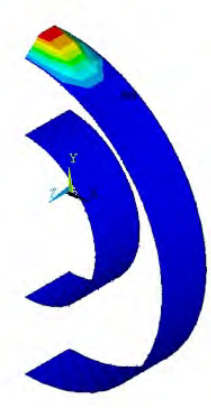

(a)

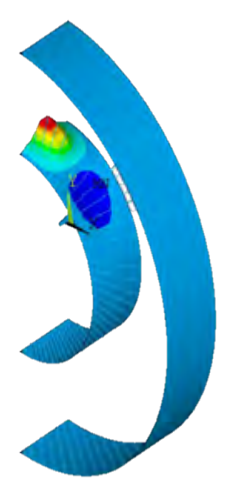

(e)

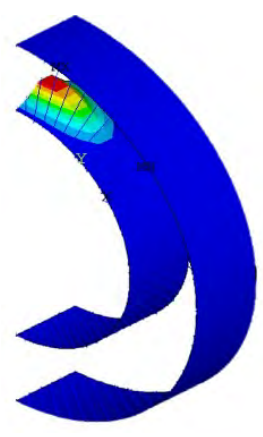

(i)

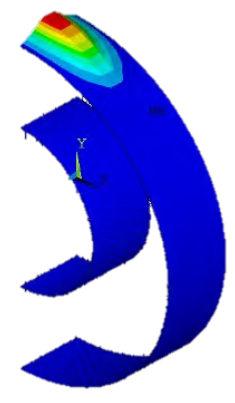

(b)

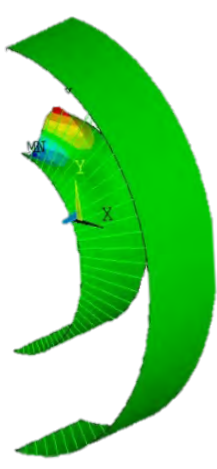

(f)

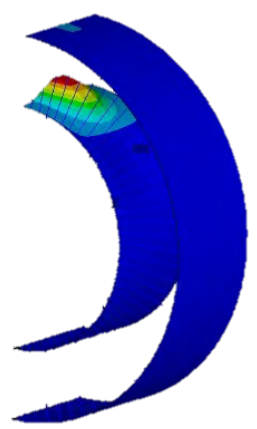

(j)

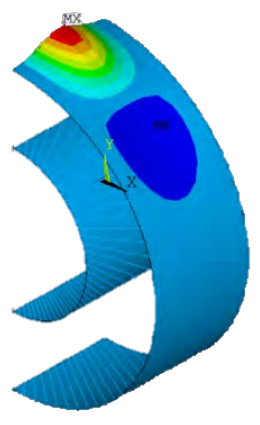

(c)

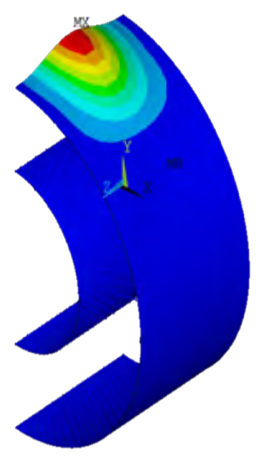

(g)

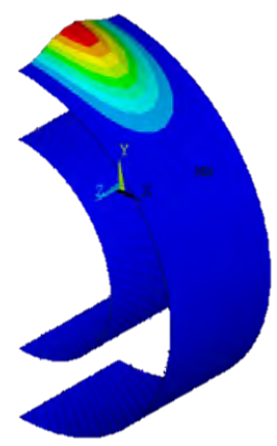

(k)

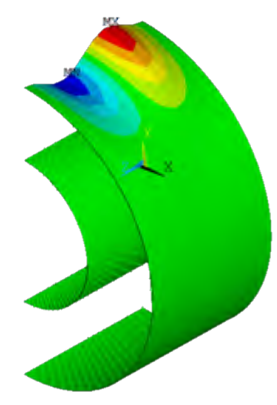

(d)

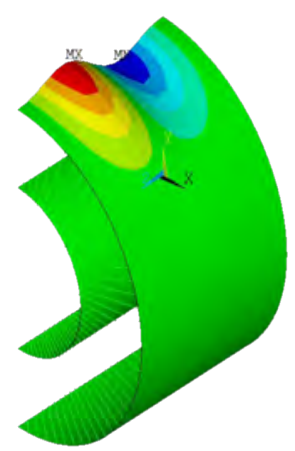

(h)

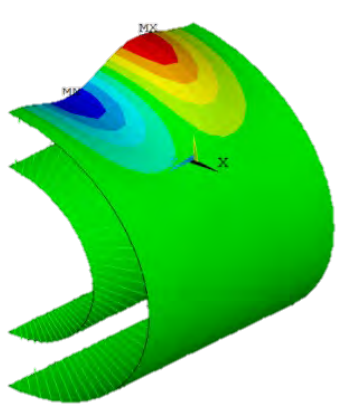

(l)

Legend

PIP-1

PIP-2

PIP-3
a) $\omega_{o}=1.40$
e) $\omega_{o}=1.10$
i) $\omega_{0}=0.99$
b) $\omega_{o}=1.68$
f) $\omega_{o}=1.86$
j) $\omega_{o}=1.66$
c) $\omega_{0}=2.80$
g) $\omega_{o}=2.92$
k) $\omega_{o}=1.98$
d) $\omega_{o}=4.02$
h) $\omega_{o}=3.98$
l) $\omega_{0}=3.95$

Figure 3 Linear elastic buckling modes for PIP-1, 2 and 3 with different lengths subjected to global bending 


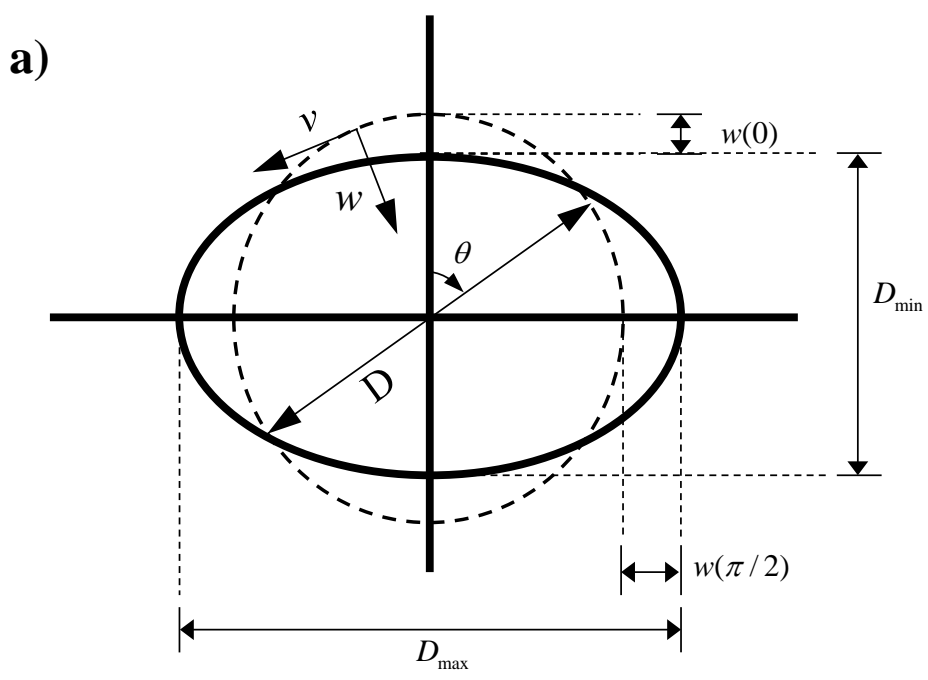

b)

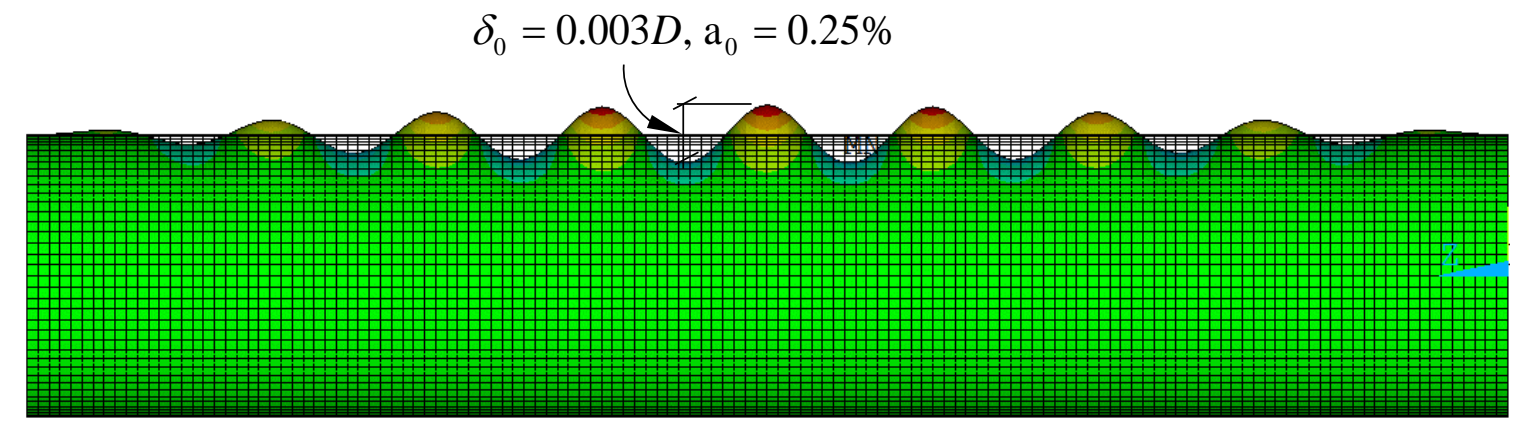

Figure 4 (a) Assumed Geometry and coordinate system to describe the ovalization of a single tube (b) Enlarged mesh of the PIP system used in the GNA, showing the imposed mesh perturbation 

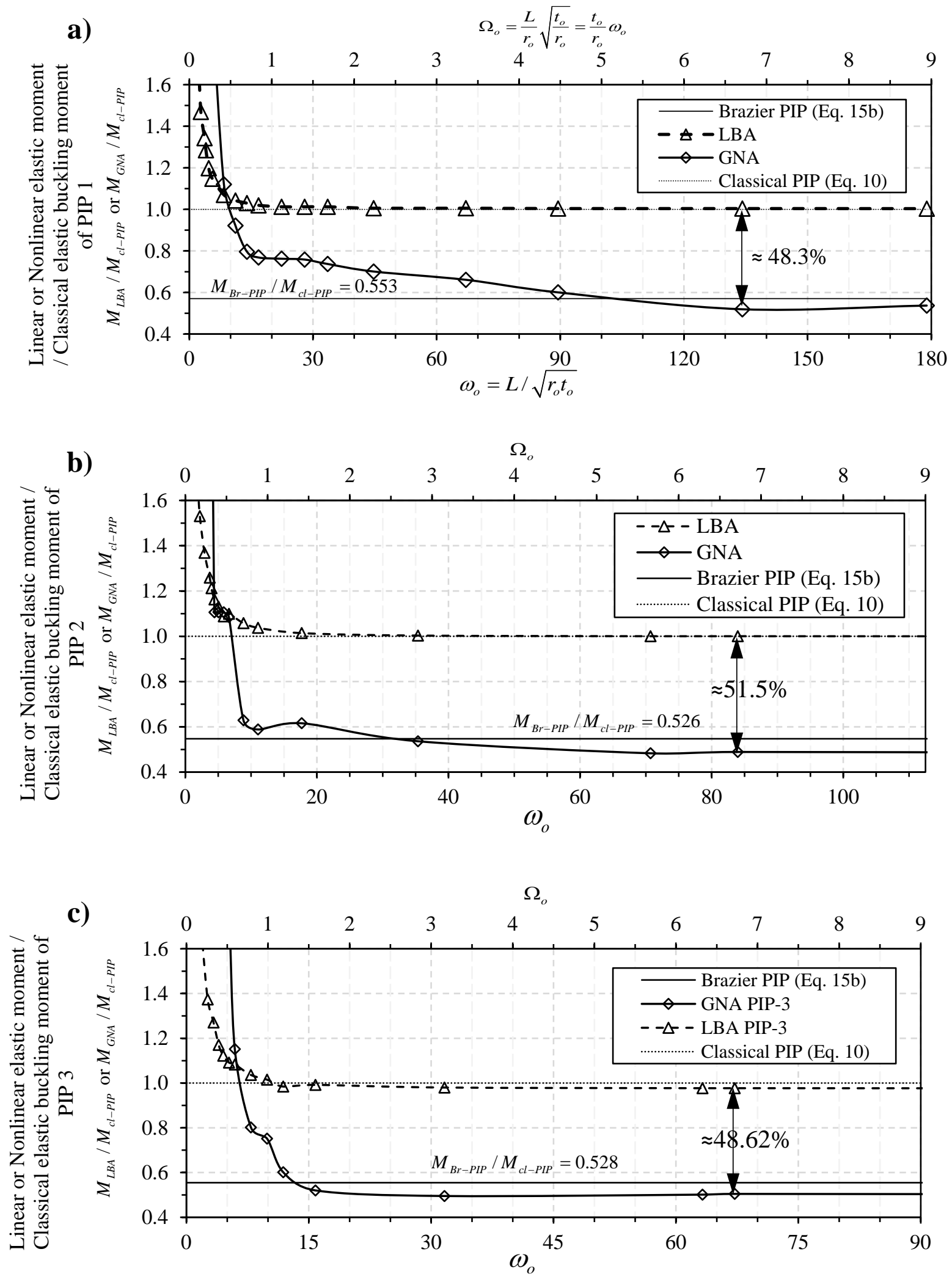

Figure 5 Influence of dimensionless length parameter on the linear (LBA) and nonlinear (GNA) elastic buckling moment of (a) PIP-1 (b) PIP-2 (c) PIP-3 


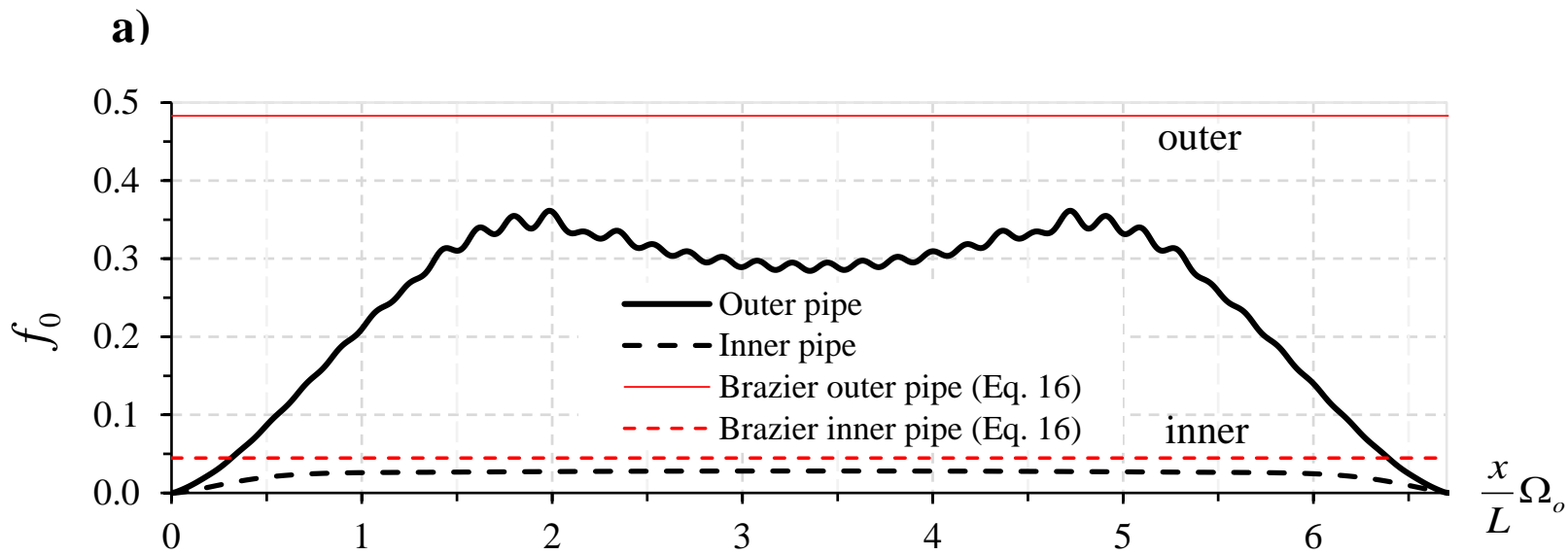

b)

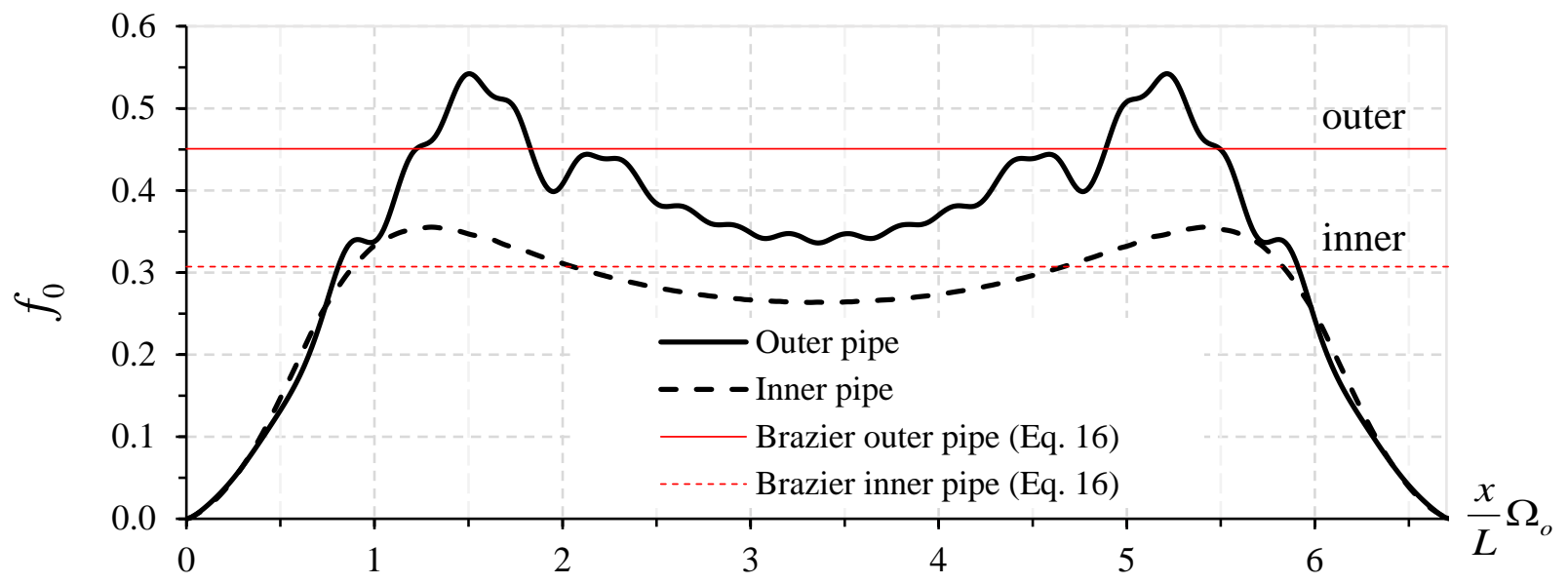

c)

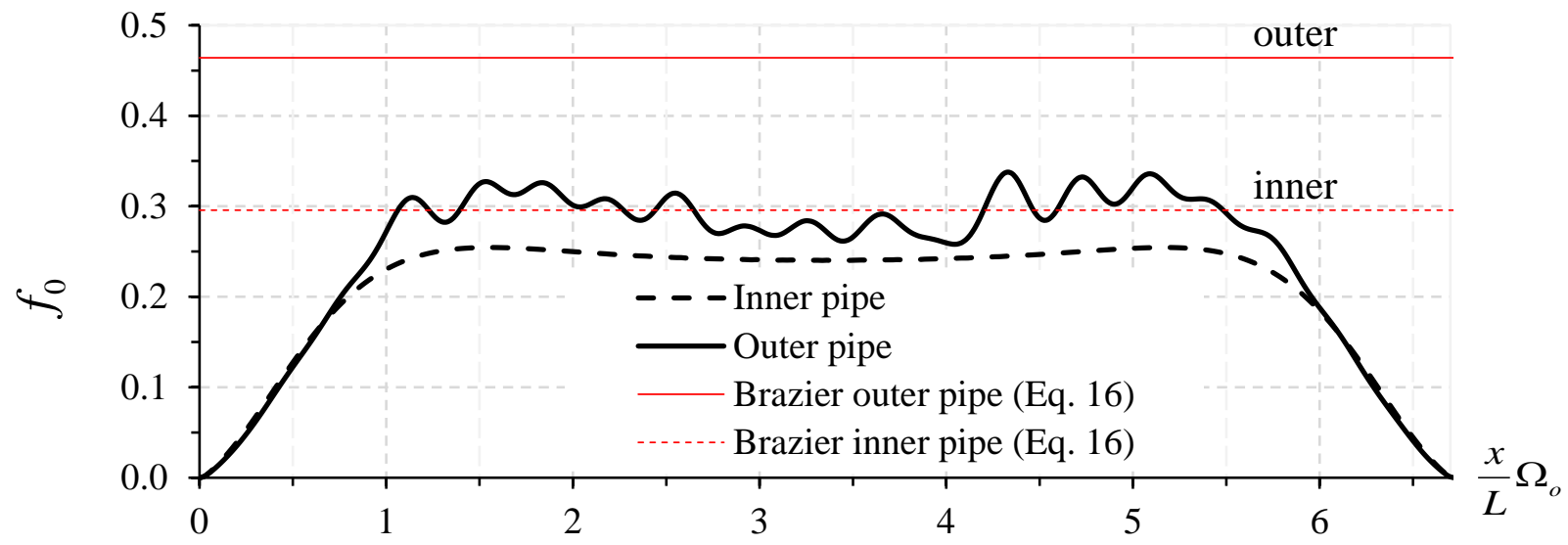

Figure 6 Ovalization along the length of (a) PIP-1 (b) PIP-2 (c) PIP-3 with $\Omega_{o}=6.7$ obtained from GNA 
a)

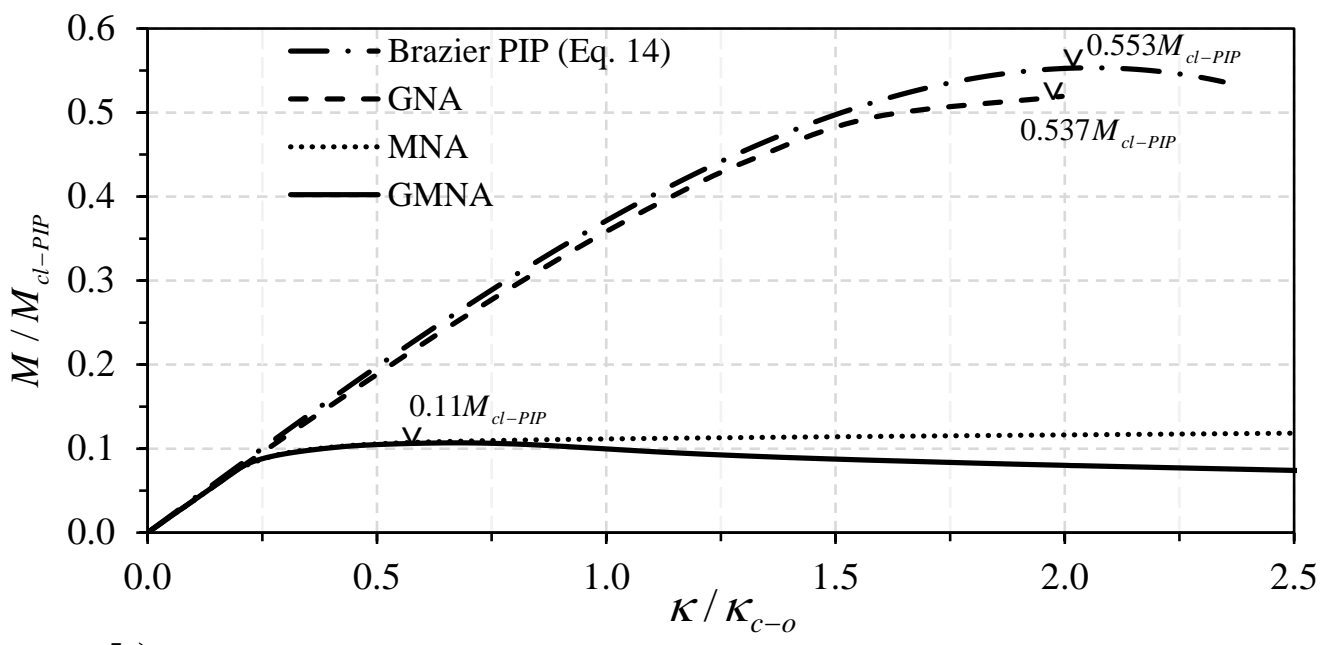

b)

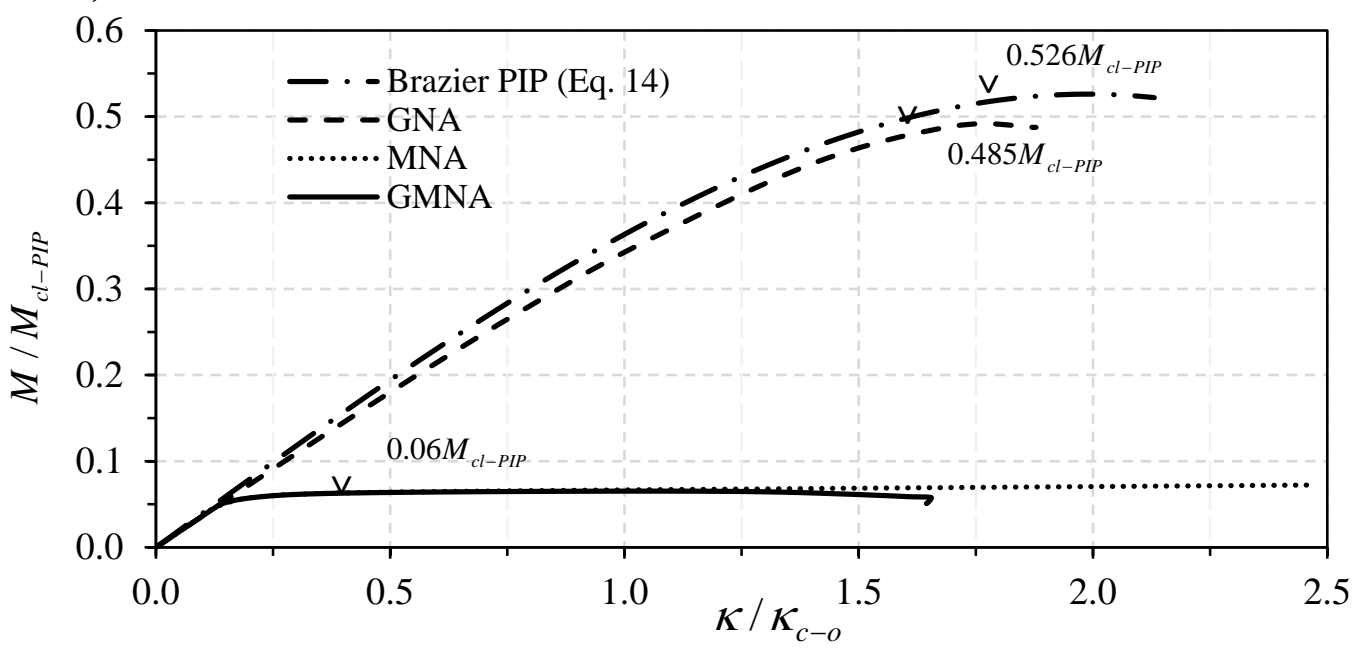

c)

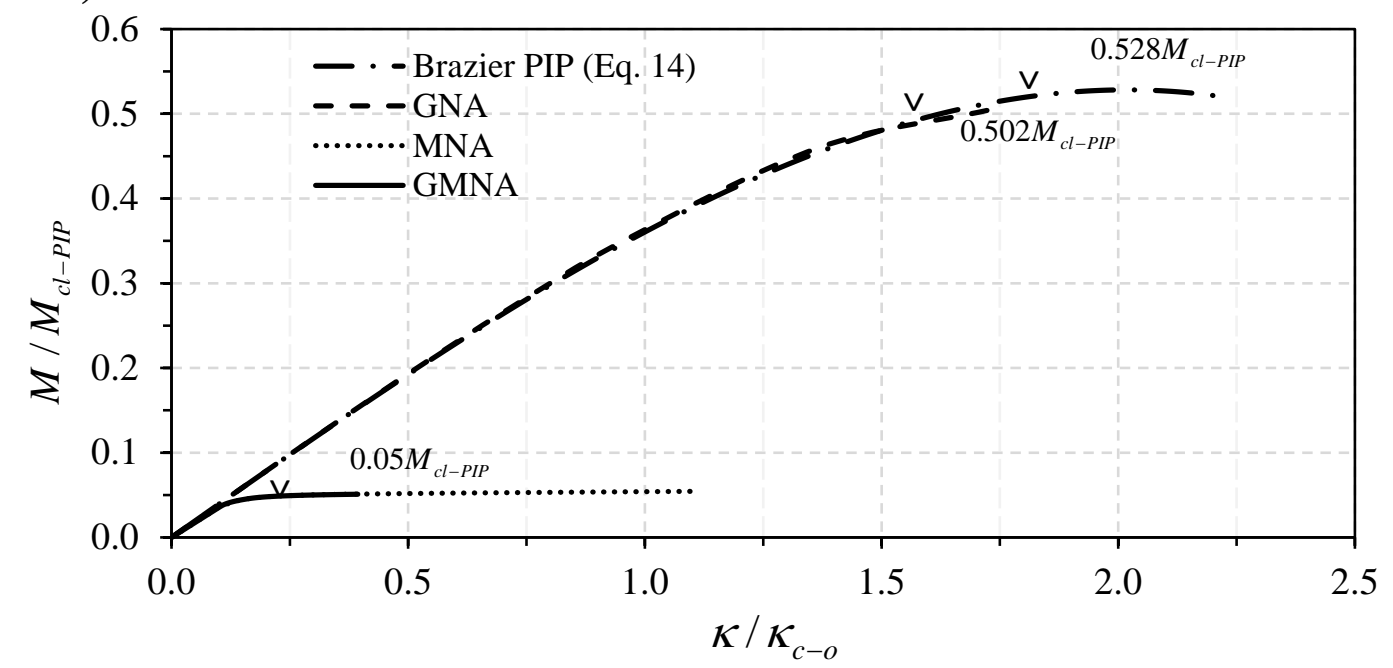

Figure 7 Normalised moment vs. normalised curvature of GNA, MNA and GMNA of (a) PIP-1 (b) PIP-2 (c) PIP-3 


\section{a)}
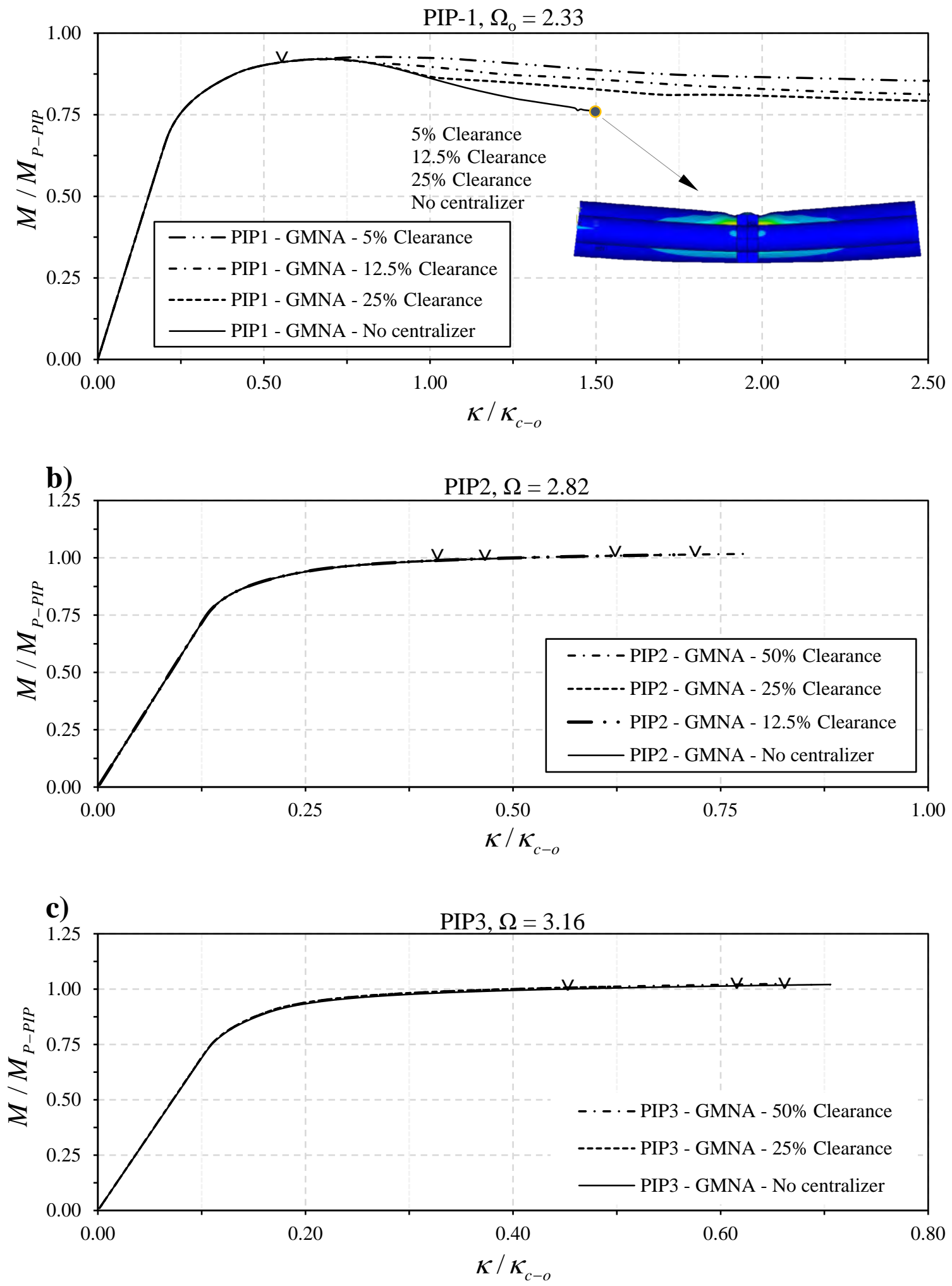

Figure $8 \mathrm{Effect}$ of centralizers with different clearances on the nonlinear geometry and nonlinear material analyses (GMNA) of (a) PIP-1 (b) PIP-2 (c) PIP-3 


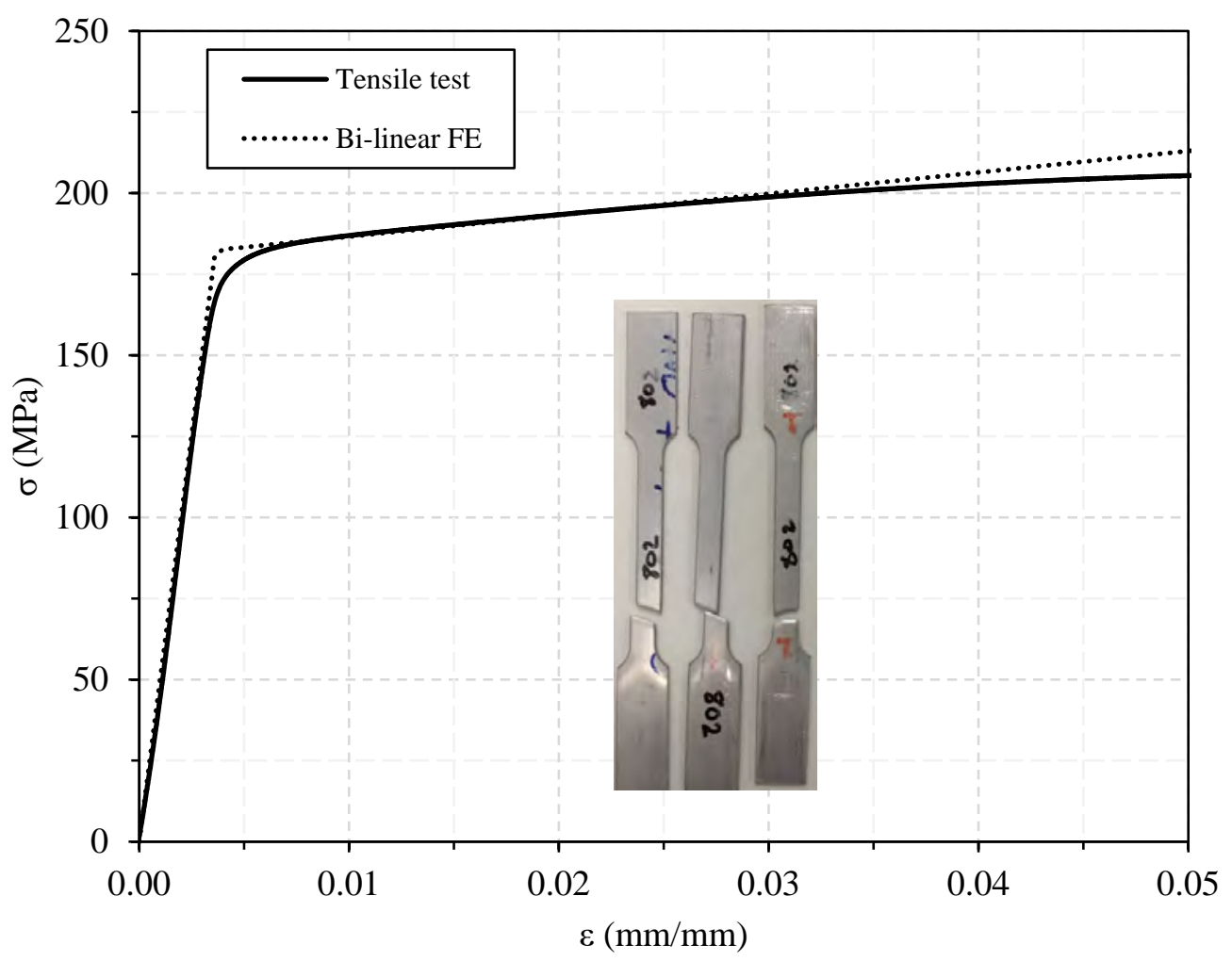

Figure 9 Stress-strain relationship of the pipe with $\mathrm{D}=80 \mathrm{~mm}$ and $\mathrm{t}=2 \mathrm{~mm}$ (outer pipe of PIP-1 in Table 4) obtained from a unidirectional tensile test and the bi linear model adopted in the FEA 
a)

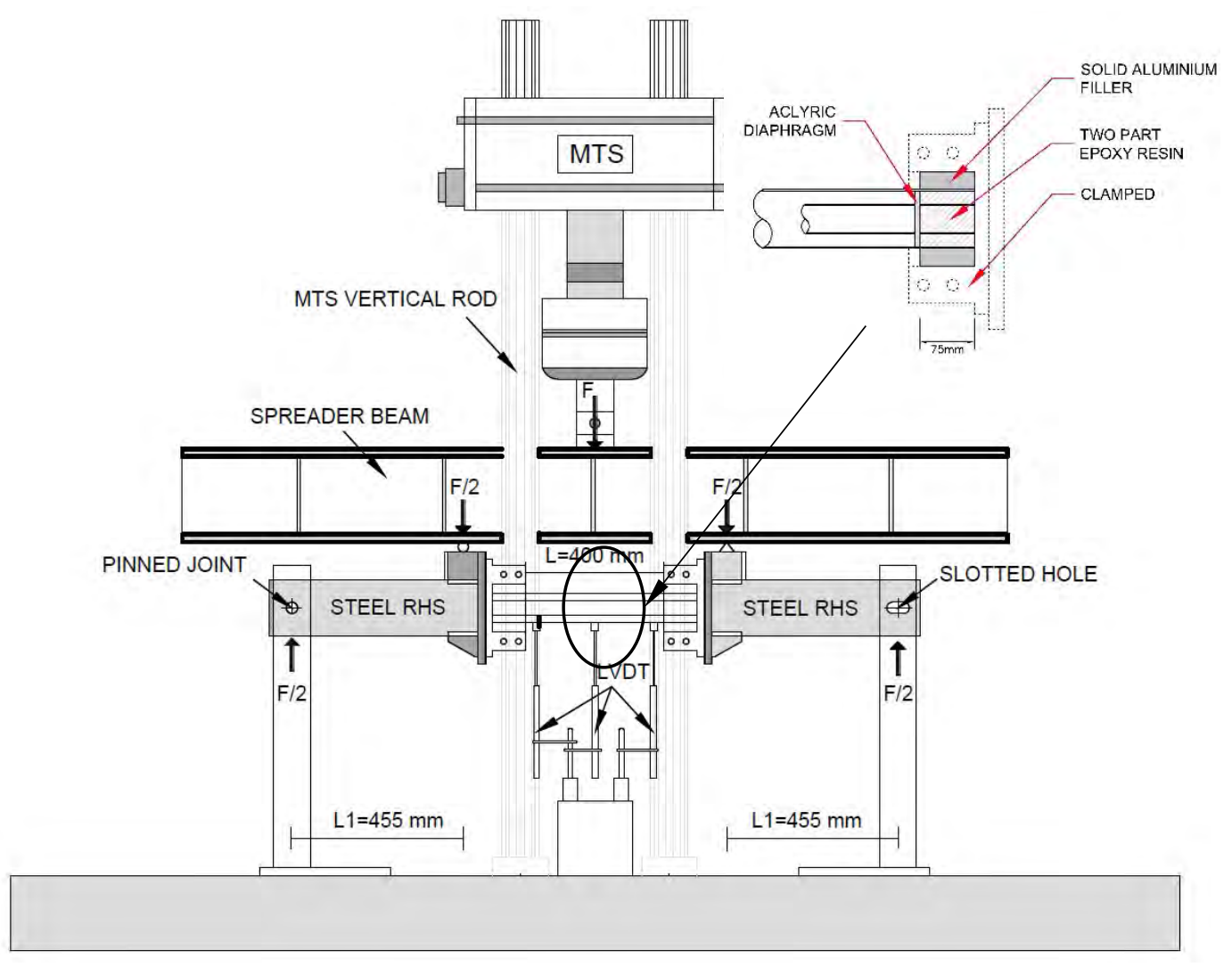

b)

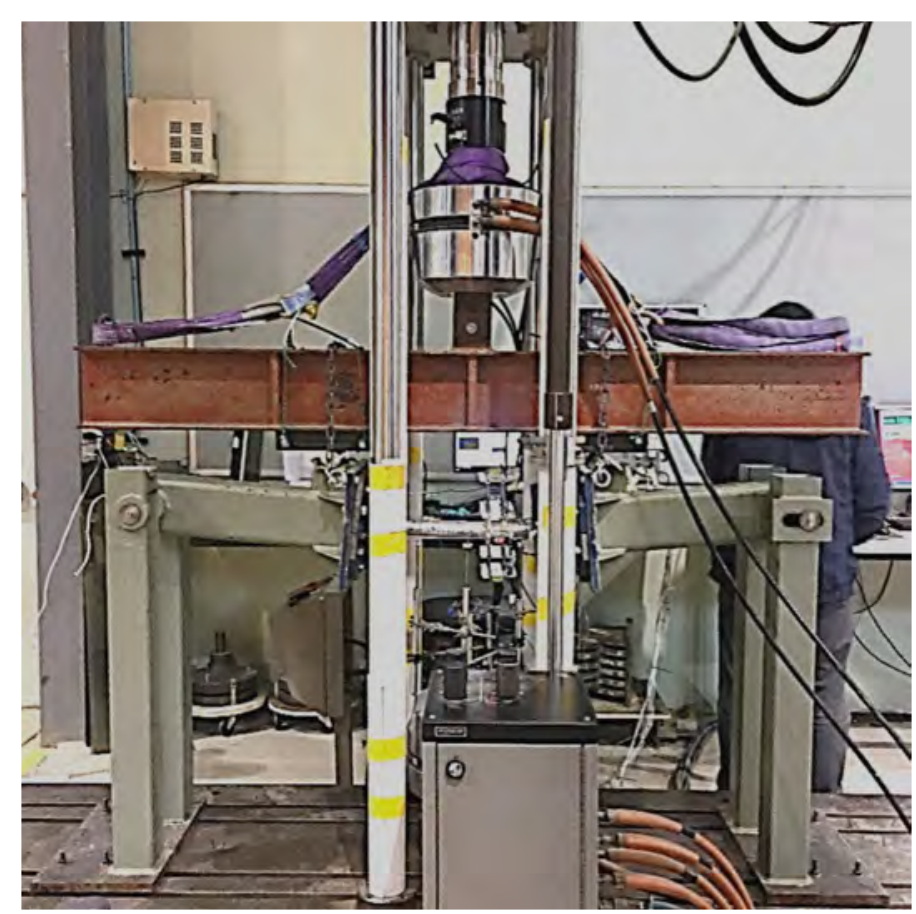

Figure 10 (a) Schematic view of the four-point bending test set-up (b) Image of four-point bending rig 
a)

b)
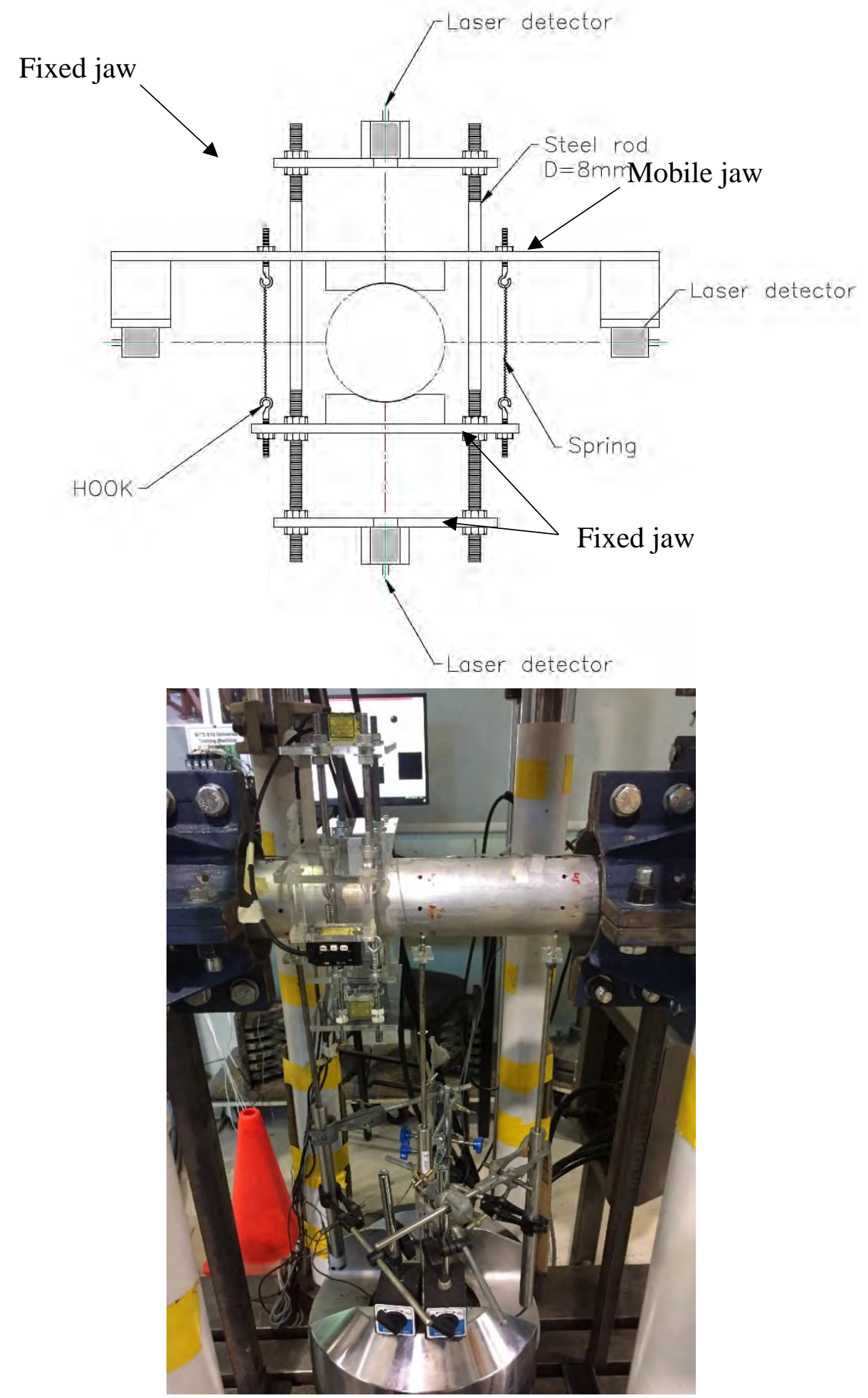

Figure 11 (a) Schematic view of the ovalization recorder used to measure ovality of the outer pipe during the bending test (b) Photo of the ovalization recorder in use 
a)

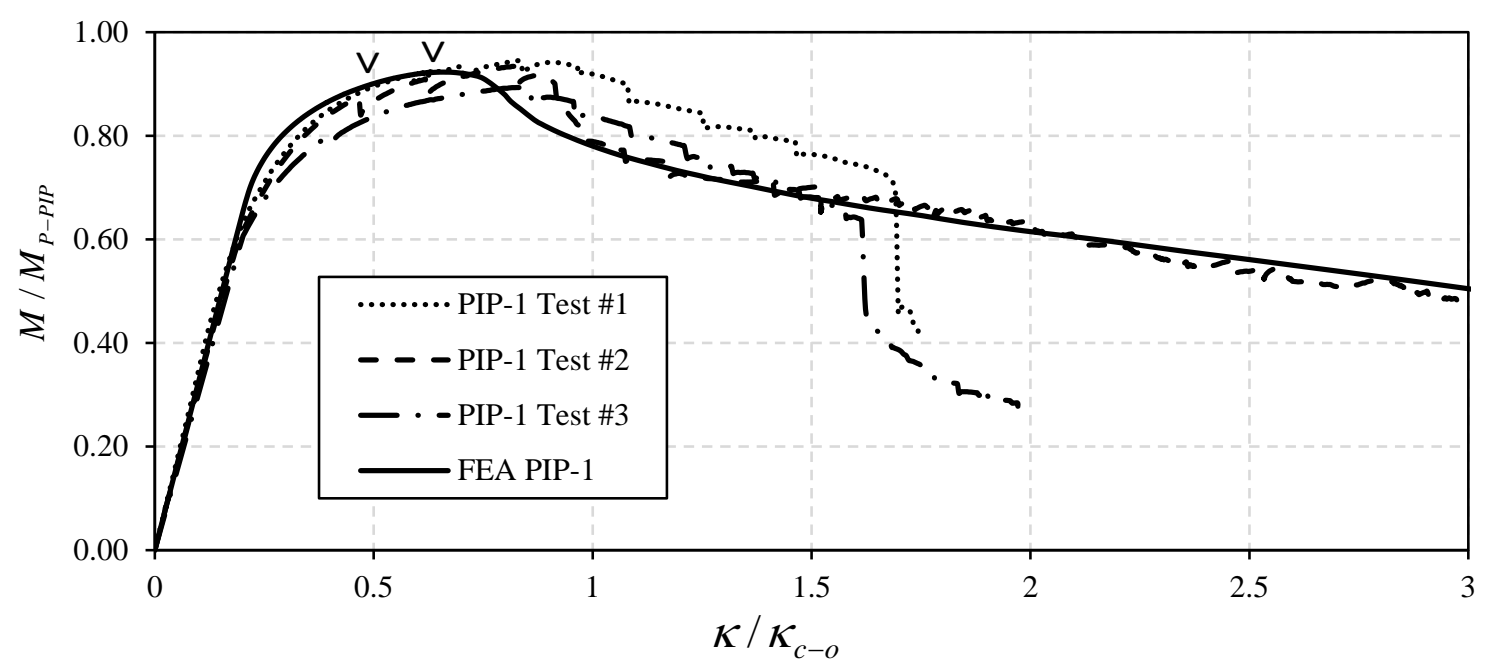

b)
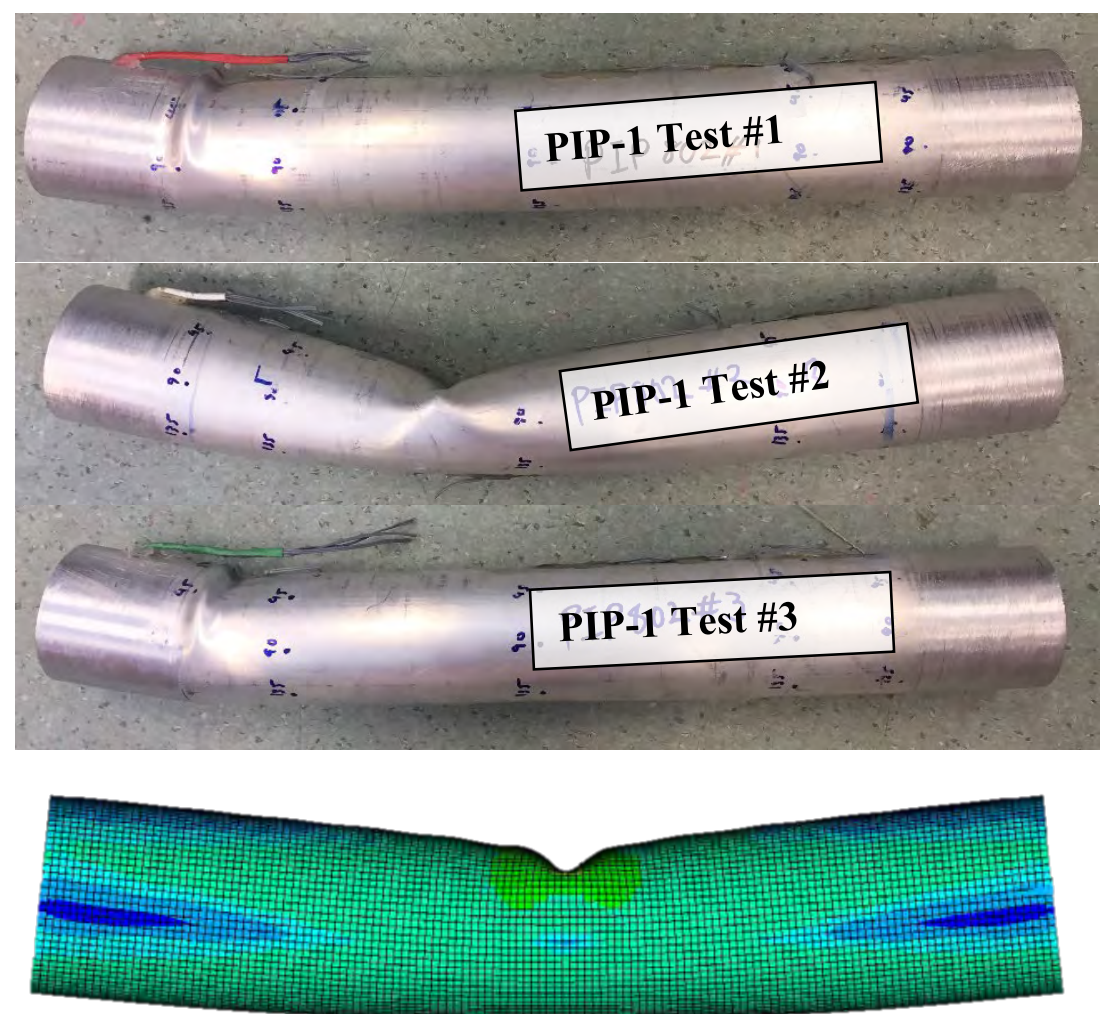

Figure 12 (a) Experimental and FEA (GMNA) bending response of PIP-1 samples in pure bending, (b) the failure modes from experiments and FEA 


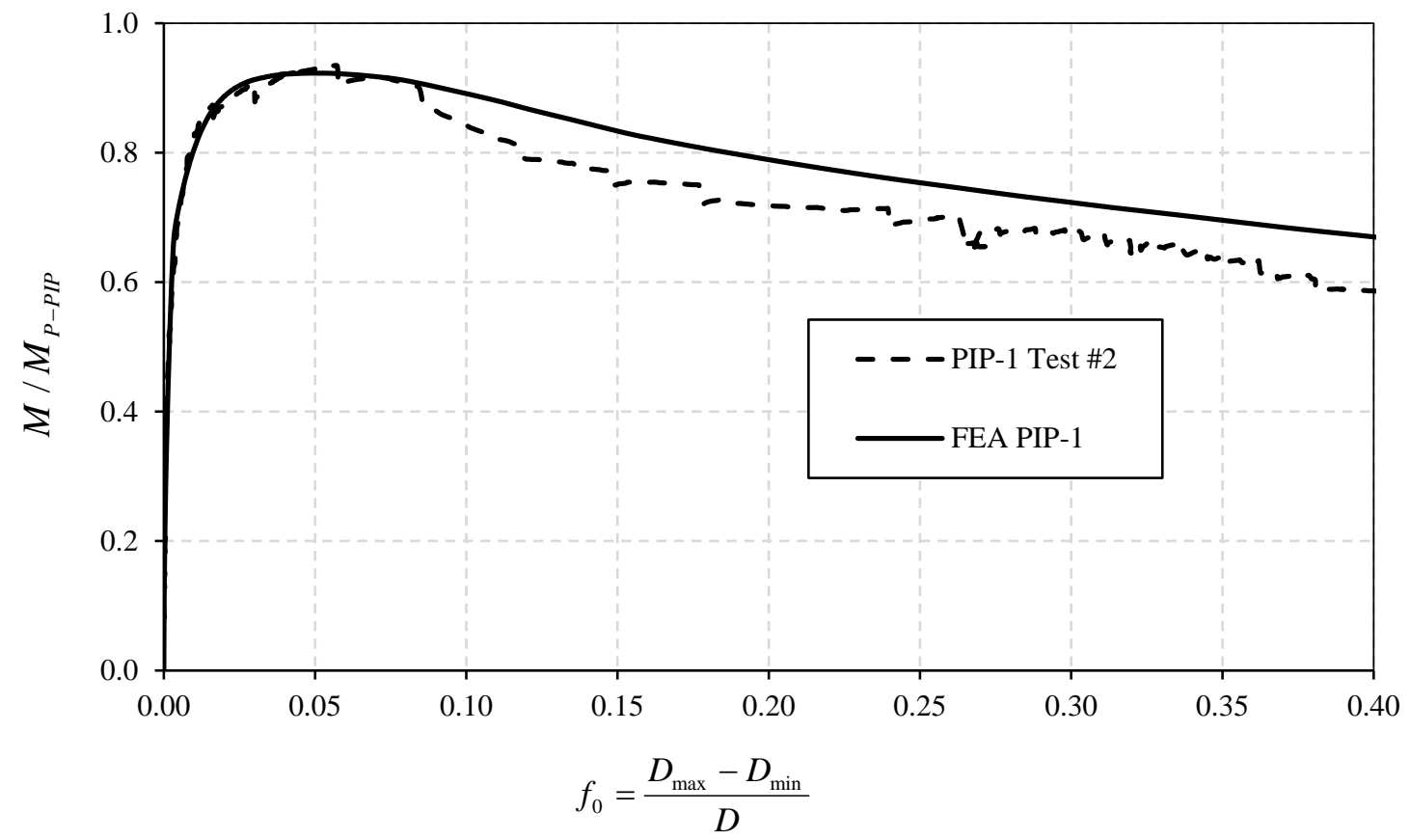

Figure 13 Normalised moment-ovalization response of the outer pipe of PIP-1 from experiment and GMNA 
a)

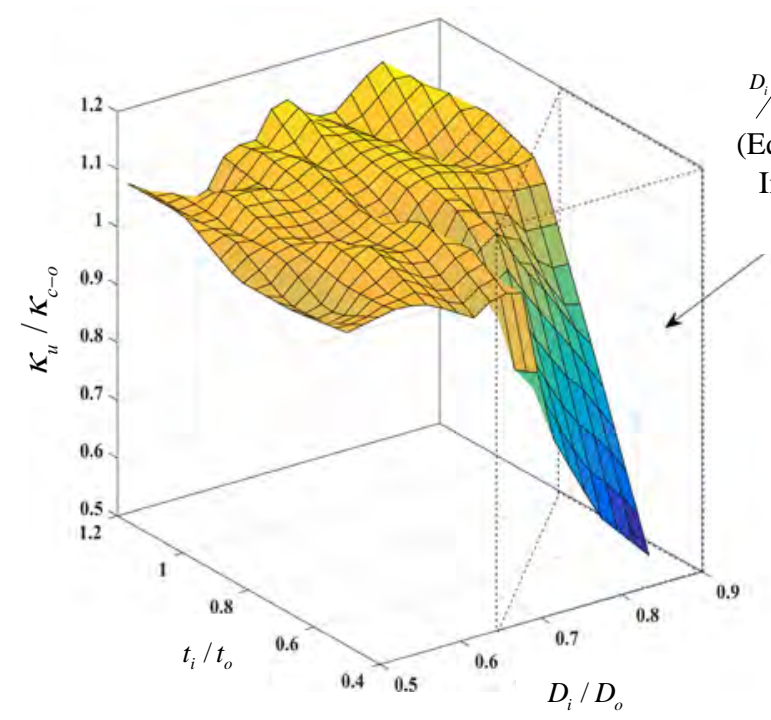

Do/to $=26.7$

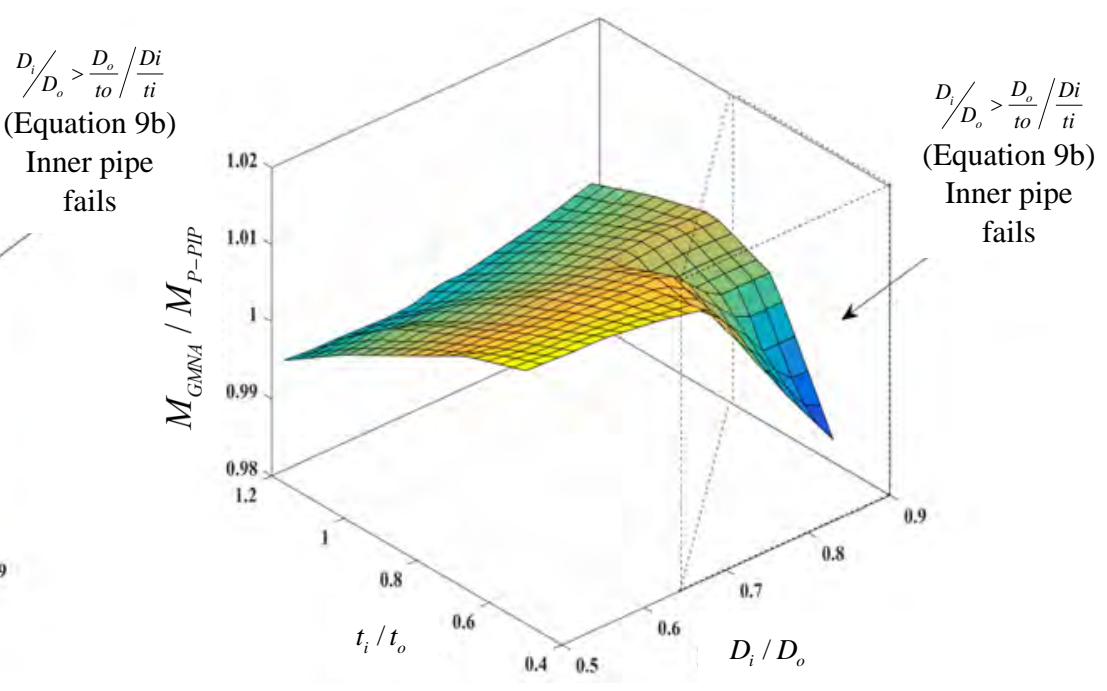

Do/to=26.7

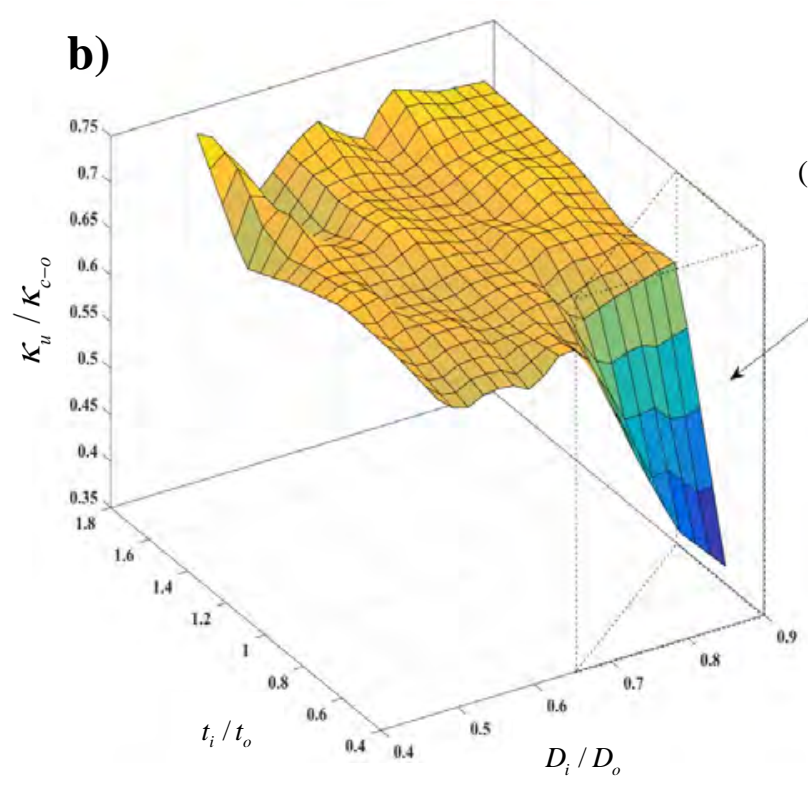

Do/to $=40$

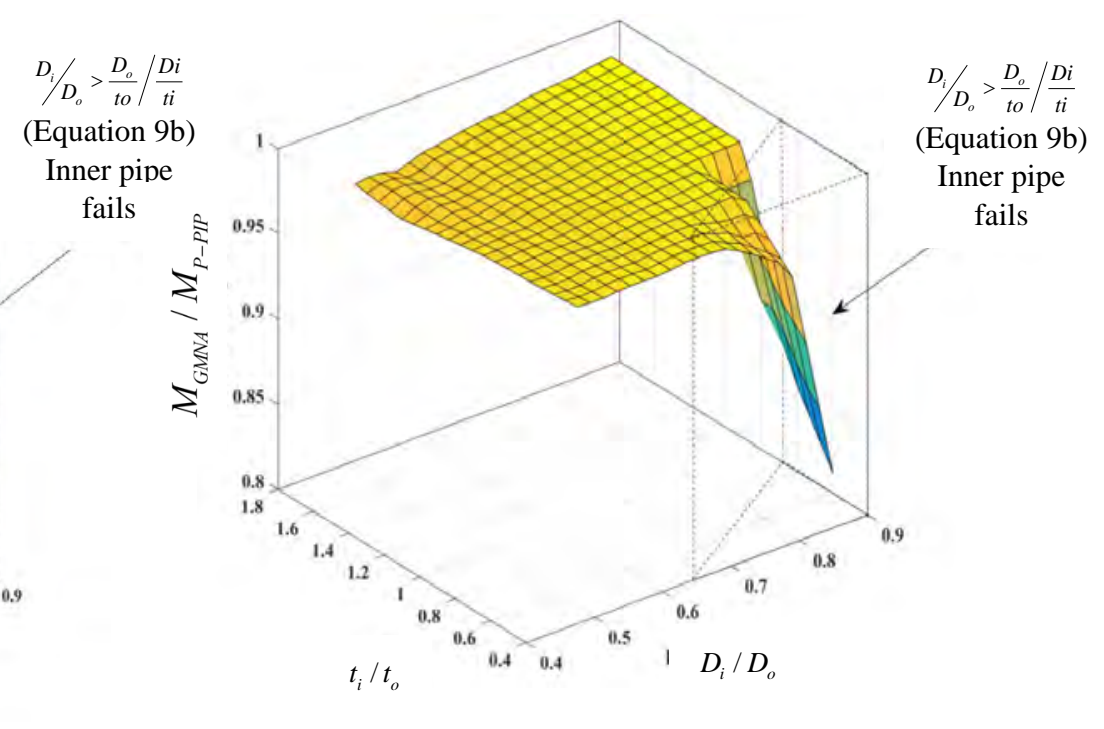

Do/to $=40$

Figure 14 Effect of $D_{i} / D_{o}$ and $t_{i} / t_{o}$ on ultimate moment and curvature capacities of (a) PIP with $D_{o} / t_{o}=26.7$ and, (b) PIP with $D_{o} / t_{o}=40$ 
a)
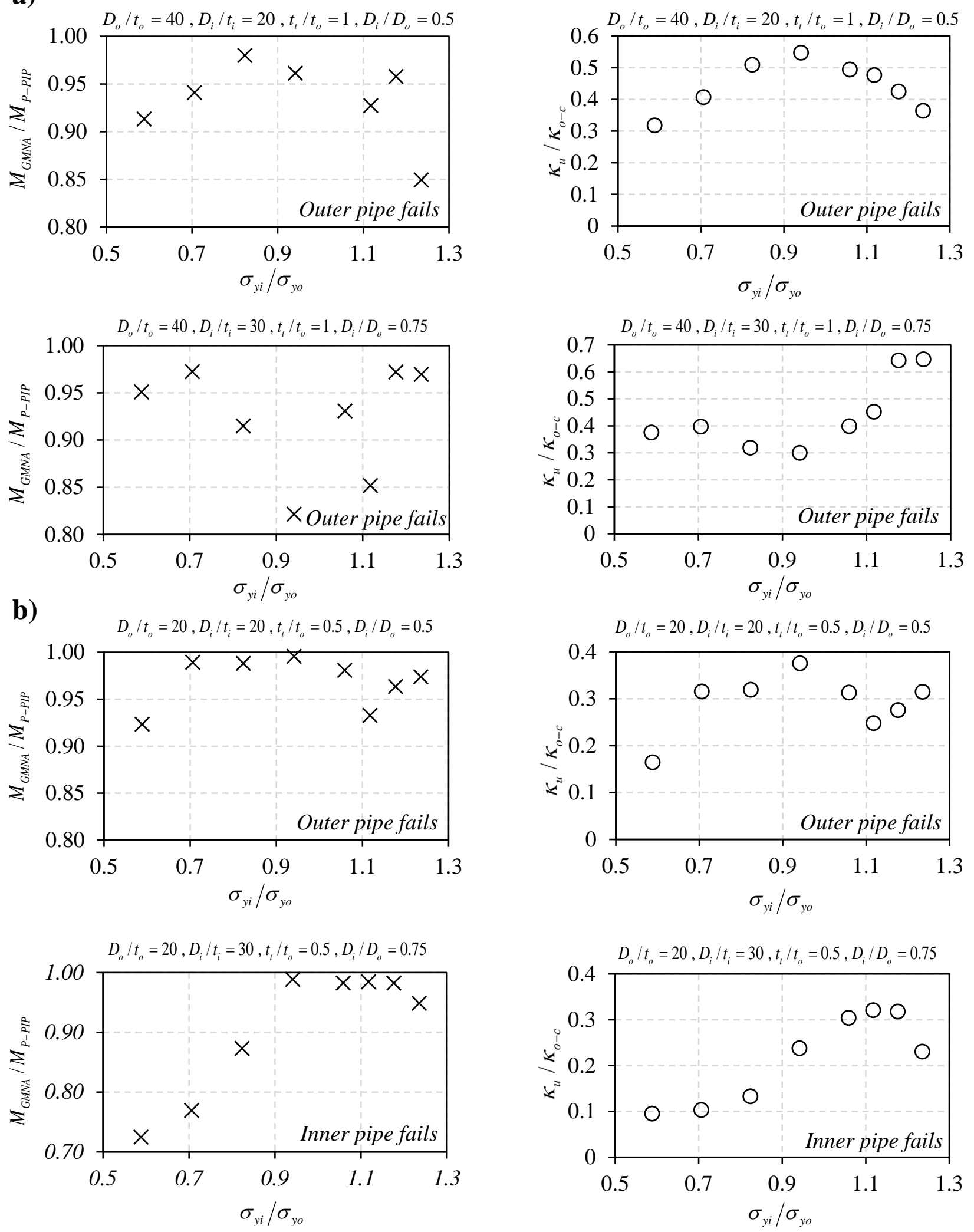

Figure 15 Effect of $\sigma_{y i} / \sigma_{y o}$ ratio on the ultimate moment capacity of PIP systems with (a) inner pipe almost as thick as the outer pipe and (b) outer pipe thinner than the inner pipe 
Table 1 Classification and characterisation of elastic isotropic cylindrical pipes in bending for $D / t \geq 40$ [19]

\begin{tabular}{lcc}
\hline Pipe class & $\omega$ range & $\Omega$ range \\
\hline Short & $3 \leq \omega<4.8$ & $\mathrm{n} / \mathrm{a}$ \\
Medium & $4.8 \leq \omega<0.5 \mathrm{r} / \mathrm{t}$ & $\Omega<0.5$ \\
& $\omega \geq 0.5 \mathrm{r} / \mathrm{t}$ & $0.5 \leq \Omega<7.0$ \\
Transitional & $\mathrm{n} / \mathrm{a}$ & $\Omega \geq 7.0$ \\
\hline
\end{tabular}

Table 2 Geometrical properties of the PIP systems used in LBA and GNA

\begin{tabular}{cccccccc}
\hline$I D$ & $D_{o} / t_{o}$ & $D_{i} / t_{i}$ & $D_{i} / D_{o}$ & $\frac{D_{o}}{t_{o}} / \frac{D_{i}}{t_{i}}$ & $\begin{array}{c}t_{o} \\
{[\mathrm{~mm}]}\end{array}$ & $\begin{array}{c}t_{i} \\
{[\mathrm{~mm}]}\end{array}$ & $v$ \\
\hline PIP-1 & 40 & 25 & 0.50 & 1.6 & 2.0 & 1.6 & 0.3 \\
PIP-2 & 25 & 40 & 0.50 & 0.6 & 3.2 & 1.0 & 0.3 \\
PIP-3 & 20 & 25 & 0.63 & 0.8 & 4.0 & 2.0 & 0.3 \\
\hline
\end{tabular}


Table 3 Comparison between the GNA, LBA and Brazier moments of long PIPs $(\Omega>7)$

\begin{tabular}{cccc}
\hline \multirow{2}{*}{$I D$} & $M_{G N A} / M_{c l-P I P}$ & $M_{G N A} / M_{\text {Br }-P I P}$ & $M_{B r-P I P} / M_{c l-P I P}$ \\
\cline { 4 - 4 } & & & Eq. 15b/Eq. 10 \\
\hline PIP-1 & 0.537 & 0.97 & 0.553 \\
PIP-2 & 0.485 & 0.92 & 0.526 \\
PIP-3 & 0.502 & 0.95 & 0.528 \\
\hline
\end{tabular}

Table 4 Effect of centralizers on limit moment and limit curvature of PIPs 1-3 (GNA)

\begin{tabular}{ccccccc}
\hline & \multicolumn{2}{c}{ without centralizer } & \multicolumn{2}{c}{ Clearance $12.5 \%$} & \multicolumn{2}{c}{ Clearance $25 \%$} \\
\cline { 2 - 6 } ID & $\frac{M_{G N A}}{M_{c l-P I P}}$ & $\kappa / \kappa_{c-o}$ & $\frac{M_{G N A}}{M_{c l-P I P}}$ & $\kappa / \kappa_{c-o}$ & $\frac{M_{G N A}}{M_{c l-P I P}}$ & $\kappa / \kappa_{c-o}$ \\
\hline PIP-1 & 0.537 & 1.950 & 0.491 & 1.497 & 0.484 & 1.509 \\
PIP-2 & 0.485 & 1.755 & 0.483 & 1.669 & 0.469 & 1.564 \\
PIP-3 & 0.502 & 1.745 & 0.464 & 1.394 & 0.460 & 1.404 \\
\hline
\end{tabular}

Table 5 Properties of the PIP-1 used in the experimental study

\begin{tabular}{lccccc}
\hline & $D[\mathrm{~mm}]$ & $D / t$ & $\begin{array}{c}E(\mathrm{GPa}) \\
(\text { Avg) }\end{array}$ & $\begin{array}{c}E^{\prime}(\mathrm{MPa}) \\
(\text { Avg) }\end{array}$ & $\begin{array}{c}\sigma_{y}(\mathrm{MPa}) \\
\text { (Avg) }\end{array}$ \\
\hline Outer pipe & 80 & 40 & 66.9 & 660 & 180 \\
Inner pipe & 40 & 25 & 65.5 & 640 & 169 \\
\hline
\end{tabular}

